Pacific Journal of Mathematics

LÉVY-HINČIN TYPE THEOREMS FOR MULTIPLICATIVE AND AR I BERCOVICI AND DAN-VIRG VOICULESC 


\title{
LÉVY-HINČIN TYPE THEOREMS FOR MULTIPLICATIVE AND ADDITIVE FREE CONVOLUTION
}

\author{
Hari Bercovici and DaN Voiculescu
}

\begin{abstract}
We give a description of infinitely divisible compactly supported probability measures relative to the multiplicative free convolutions on the positive half-line and on the unit circle. A new proof is provided for the analogous result for additive free convolution.
\end{abstract}

1. Introduction. In classical probability theory an important role is played by the convolution of probability distributions on the real line; indeed, the distribution of the sum of two independent random variables is the convolution of the distributions of the two summands. In connection with this, the infinitely divisible probability distributions, and the associated convolution semigroups, occupy a central place. On the one hand, infinitely divisible probability distributions are the natural object in the study of the limits of sums of independent random variables, and on the other, convolution semigroups are related with stationary processes with independent increments; see [2, 3, and 4] for further information in the classical situation. The Lévy-Hinčin formula gives a complete description of all the infinitely divisible distributions on the real line, and it shows that such distributions can be obtained as limits of convolutions of Gaussian and Poisson distributions.

In this paper we study infinite divisibility for multiplicative and additive free convolution, which are two operations arising from the non-commutative probability theory of free products; see $[5,6,7$, 8, and 9] for the background of this non-commutative theory. We will describe briefly the definition of the free convolution operations. Consider a unital algebra $A$, endowed with a functional $\phi$ such that $\phi(1)=1$; the elements $x \in A$ will be called random variables. One can associate with each random variable $x$ an analytic functional $\nu_{x}$, i.e., a functional on the polynomial algebra $\mathbf{C}[X]$, by the formula $\nu_{x}(p)=\phi(p(x))$ if $p \in \mathbf{C}[X]$. If $x$ and $y$ are two free random variables (in a technical sense which we will not explain here) then $\nu_{x+y}$ and $\nu_{x y}$ can be shown to depend only on $\nu_{x}$ and $\nu_{y}$. This allows one to define the additive and multiplicative free convolution 
of analytic functionals by $\nu_{x} \boxplus \nu_{y}=\nu_{x+y}$ and $\nu_{x} \otimes \nu_{y}=\nu_{x y}$. Now, given a compactly supported measure $\mu$ in the complex plane, one can regard it as an analytic functional by setting $\mu(p)=\int_{\mathbf{C}} p(z) d \mu(z)$ if $p \in \mathbf{C}[X]$. Moreover, the measure $\mu$ is completely determined by the corresponding analytic functional if the support of $\mu$ is contained in the real line $\mathbf{R}$ or the unit circle $\mathbf{T}$. The following observations help then define the additive and multiplicative convolution operations on certain classes of compactly supported measures.

(1) If $\mu$ and $\nu$ are compactly supported probability measures on $\mathbf{R}$, then $\mu \boxplus \nu$ is also a compactly supported probability measure on $\mathbf{R}$.

(2) If $\mu$ and $\nu$ are compactly supported probability measures on $\mathbf{T}$, then $\mu \otimes \nu$ is also a compactly supported probability measure on $\mathbf{T}$.

(3) If $\mu$ and $\nu$ are compactly supported probability measures on the positive axis $\mathbf{R}_{+}=(0,+\infty)$, then $\mu \otimes \nu$ is also a compactly supported probability measure on $\mathbf{R}_{+}$.

Infinite divisibility for free additive convolution was studied in [6]. A complete characterization of $\boxplus$-infinitely divisible distributions was given, and it was explained in [9] that this characterization is an analogue of the classical Lévy-Hinčin characterization. In fact, the analogue of the Gaussian distribution appeared even earlier, when the central limit theorem for free additive convolution was proved in [5].

The present paper grew out of a desire to obtain similar results for the $\otimes$-infinitely divisible probability distributions supported on $\mathbf{R}_{+}$ or on the unit circle $\mathbf{T}$. While working on this project, we realized that the methods required in the multiplicative case can also be used in the additive case, and they yield a substantial simplification of the argument used in [6]. This simplification is described in $\S 8$ below, which is substantially self-contained. In fact we show that the LévyHinčin measure associated with a $\boxplus$-infinitely divisible distribution $\mu$ can be calculated, as in the classical case, as a weak limit of measures related with the convolution semigroup generated by $\mu$.

Let us denote by $\mathscr{M}$ the set of all compactly supported Borel probability measures $\mu$ on the complex plane $\mathbf{C}$. We denote by $\mathscr{M}_{\mathbf{R}}, \mathscr{M}_{\mathbf{R}_{+}}$, and $\mathscr{M}_{\mathrm{T}}$ the set of those measures $\mu \in \mathscr{M}$ which are supported by $\mathbf{R}$, $\mathbf{R}_{+}$, and T, respectively. We also set $\mathscr{M}_{*}=\left\{\mu \in \mathscr{M}: \int_{\mathbf{C}} z d \mu(z) \neq 0\right\}$. We note for further reference the following properties of free convolutions:

(1) $\left(\mathscr{M}_{\mathbf{R}}, \boxplus\right)$ is a commutative semigroup; 
(2) $\left(\mathscr{M}_{\mathbf{R}_{+}}, \nabla\right)$ is a commutative semigroup;

(3) $\left(\mathscr{M}_{\mathrm{T}}^{+}, \otimes\right)$ is a commutative semigroup;

(4) $\mathscr{M}_{\mathbf{R}_{+}} \subset \mathscr{M}_{*}$;

(5) $\left(\mathscr{M}_{\mathrm{T}}^{+} \cap \mathscr{M}_{*}, \otimes\right)$ is a commutative semigroup;

(6) if $\mu_{1}, \mu_{2} \in \mathscr{M}_{\mathbf{T}}$ and $\mu_{2} \notin \mathscr{M}_{*}$, then $\int_{\mathbf{T}} z^{n} d \mu_{1} \otimes \mu_{2}(z)=0$ for $n=1,2, \ldots$.

As mentioned above, we will study infinitely divisible probability measures relative to free convolution. More precisely, an element $\mu \in \mathscr{M}$ is said to be $\otimes$-infinitely divisible if for every natural number $n$ there exists a measure $\mu_{n} \in \mathscr{M}$ such that

$$
\mu=\underbrace{\mu_{n} \otimes \mu_{n} \otimes \cdots \otimes \mu_{n}}_{n \text { times }} .
$$

Of course, we have to restrict ourselves to measures in $\mathscr{M}_{\mathbf{R}_{+}}$or $\mathscr{M}_{\mathbf{T}}$, so that the free convolution is defined. The main tool that we use is a result of [7] that gives a function theoretical property of the free multiplicative convolution. More precisely, it is shown in [7] that one can associate with each $\mu \in \mathscr{M}_{*}$ a convergent power series $S_{\mu}(z)$ such that $S_{\mu}(z)=S_{\mu_{1}}(z) S_{\mu_{2}}(z)$ if $\mu, \mu_{1}, \mu_{2} \in \mathscr{M}_{*}$ and $\mu=\mu_{1} \otimes \mu_{2}$. Our characterizations of $\otimes$-infinitely divisible probability measures in $\mathscr{M}_{\mathbf{R}_{+}}$ and $\mathscr{M}_{\mathrm{T}}$ will be given in terms of the corresponding functions $S$.

The paper is organized as follows. Sections 2 and 3 contain a detailed discussion of the functions $S_{\mu}$ for $\mu \in \mathscr{M}_{\mathrm{T}} \cap \mathscr{M}_{*}$ and $\mu \in \mathscr{M}_{\mathbf{R}_{+}}$, respectively. Section 4 contains some calculations of functions $S_{\mu}$ for simple measures $\mu$. These calculations are useful later in the calculation of analogues of the Gaussian and Poisson distributions. In $\S 5$ we give a limit theorem for the description of the function $S_{\mu}$ when $\mu$ belongs to a $\Delta$-convolution semigroup. Sections 6 and 7 provide the analogues of the Lévy-Hinčin formula for measures in $\mathscr{M}_{\mathrm{T}} \cap \mathscr{M}_{*}$ and $\mathscr{M}_{\mathbf{R}_{+}}$, respectively. Finally, $\S 8$ is dedicated to an application of the methods of the paper to $\boxplus$-infinitely divisible distributions.

Our main reference for the theory of moments and Nevanlinna-Pick interpolation is Achieser [1]. For background in probability theory, like the classical theory of infinitely divisible distributions and the Lévy-Hinčin formula, we refer to [2, 3, and 4].

2. The function $S_{\mu}$ for $\mu \in \mathscr{M}_{\mathrm{T}} \cap \mathscr{M}_{*}$. We recall for convenience the construction of the function $S_{\mu}$ for $\mu \in \mathscr{M}_{*}$. First one considers the function

$$
\psi_{\mu}(z)=\int_{\mathbf{C}} \frac{z t}{1-z t} d \mu(t)
$$


which is defined and analytic in a neighborhood of $z=0$. Moreover, $\psi_{\mu}(0)=0$ and $\psi_{\mu}^{\prime}(0)=\int_{\mathbf{C}} t d \mu(t) \neq 0$ since $\mu \in \mathscr{M}_{*}$. It follows that there exists a function $\chi_{\mu}(z)$, analytic in a neighborhood of zero, such that

$$
\psi_{\mu}\left(\chi_{\mu}(z)\right)=\chi_{\mu}\left(\psi_{\mu}(z)\right)=z
$$

for sufficiently small $z$. Clearly $\chi_{\mu}(0)=0$ and $\chi_{\mu}^{\prime}(0)=1 / \psi_{\mu}^{\prime}(0)$, and hence the function

$$
S_{\mu}(z)=\frac{z+1}{z} \chi_{\mu}(z)
$$

is also analytic in a neighborhood of $z=0$, and $S_{\mu}(0)=\chi_{\mu}^{\prime}(0) \neq 0$.

Sometimes it will be convenient to use the function

$$
\Sigma_{\mu}(z)=S_{\mu}\left(\frac{z}{1-z}\right),
$$

which is also analytic in a neighborhood of zero. If we introduce the functions

$$
\psi_{\mu}^{\sim}(z)=\frac{\psi_{\mu}(z)}{1+\psi_{\mu}(z)}, \quad \chi_{\mu}^{\sim}(z)=\chi\left(\frac{z}{1-z}\right),
$$

then

$$
\psi_{\mu}^{\sim}\left(\chi_{\mu}^{\sim}(z)\right)=\chi_{\mu}^{\sim}\left(\psi_{\mu}^{\sim}(z)\right)=z
$$

for $z$ close to zero, and $\Sigma_{\mu}(z)=(1 / z) \chi_{\mu}^{\sim}(z)$.

2.1. Lemma. Assume that $\mu_{1}, \mu_{2} \in \mathscr{M}_{*}$ and $S_{\mu_{1}}=S_{\mu_{2}}$. If either $\mu_{1}, \mu_{2} \in \mathscr{M}_{\mathbf{R}_{+}}$or $\mu_{1}, \mu_{2} \in \mathscr{M}_{\mathbf{T}}$, then $\mu_{1}=\mu_{2}$.

Proof. The condition $S_{\mu_{1}}=S_{\mu_{2}}$ implies, of course $\psi_{\mu_{1}}=\psi_{\mu_{2}}$ or, equivalently,

$$
\int_{\mathbf{C}} z^{n} d \mu_{1}(z)=\int_{\mathbf{C}} z^{n} d \mu_{2}(z), \quad n=1,2, \ldots
$$

We also have then

$$
\int_{\mathbf{C}} \bar{z}^{n} d \mu_{1}(z)=\int_{\mathbf{C}} \bar{z}^{n} d \mu_{2}(z), \quad n=1,2, \ldots,
$$

and $\int_{\mathbf{C}} d \mu_{1}(z)=\int_{\mathbf{C}} d \mu_{2}(z)=1$. If both $\mu_{1}$ and $\mu_{2}$ are supported on $\mathbf{R}_{+}$or $\mathbf{T}$ then the corresponding Weierstrass approximation theorem implies immediately that $\mu_{1}=\mu_{2}$.

It is important to note that the equality $S_{\mu_{1}}=S_{\mu_{2}}$ does not always imply $\mu_{1}=\mu_{2}$. The following example is relevant in this direction. 
Fix a point $\alpha \in \mathbf{C},|\alpha|<1$, and denote $\mu_{1}=\delta_{\alpha}$ (Dirac unit mass at $\alpha)$. Define next $\mu_{2}=P_{\alpha}$ on $\mathbf{T}=\left\{e^{i t}: 0 \leq t<2 \pi\right\}$ by

$$
d \mu_{2}=d P_{\alpha}=\frac{1}{2 \pi} \frac{1-|\alpha|^{2}}{\left|\alpha-e^{i t}\right|^{2}} d t .
$$

Clearly $d \mu_{2}$ is harmonic measure for the unit disc, relative to the point $\alpha$, and therefore $\int_{\mathbf{C}} z^{n} d \mu_{1}(z)=\alpha^{n}=\int_{\mathbf{C}} z^{n} d \mu_{2}(z), \quad n=1,2, \ldots$. If $\alpha \neq 0$ then $\mu_{1}, \mu_{2} \in \mathscr{M}_{*}$ and $S_{\mu_{1}}=S_{\mu_{2}}$. Using dominating sequences in the unit disc one can in fact construct purely atomic measures $\mu_{1} \neq \mu_{2}$ such that $S_{\mu_{1}}=S_{\mu_{2}}$.

We proceed now to give descriptions of the functions $S_{\mu}$ and $\Sigma_{\mu}$ associated with measures $\mu \in \mathscr{M}_{\mathrm{T}} \cap \mathscr{M}_{*}$.

2.2. Proposition. Let $S(z)$ be a convergent power series. Then $S=S_{\mu}$ for some $\mu \in \mathscr{M}_{\mathrm{T}} \cap \mathscr{M}_{*}$ if and only if the following conditions are satisfied:

(1) $|S(0)| \geq 1$;

(2) there exists $\varepsilon>0$ such that, given a natural number $n$ and points $z_{1}, z_{2}, \ldots, z_{n} \in \mathbf{C}$ with $\left|z_{j}\right|<\varepsilon, j=1,2, \ldots, n$, the matrix

$$
\left[\frac{z_{j}+\bar{z}_{k}+1}{1-\frac{z_{j} S\left(z_{j}\right)}{1+z_{j}} \frac{\overline{z_{k} S\left(z_{k}\right)}}{1+\bar{z}_{k}}}\right]_{j, k=1}^{n}
$$

is nonnegative definite.

If $S=S_{\mu}$ then the number $\varepsilon$ can be chosen to be the greatest with the property that $\left|\chi_{\mu}(z)\right|<1$ for $|z|<\varepsilon$.

Proof. Let $\mu \in \mathscr{M}_{\mathrm{T}} \cap \mathscr{M}_{*}$ and note that $S_{\mu}(0)=\chi_{\mu}^{\prime}(0)=1 / \psi_{\mu}^{\prime}(0)$, and

$$
\left|\psi_{\mu}^{\prime}(0)\right|=\left|\int_{\mathbf{T}} \zeta d \mu(\zeta)\right| \leq \int_{\mathbf{T}}|\zeta| d \mu(\zeta)=1 .
$$

This proves the necessity of (1). Observe next that

$$
\psi_{\mu}(z)=\int_{\mathbf{T}} \frac{z \zeta}{1-z \zeta} d \mu(\zeta)=-\frac{1}{2}+\frac{1}{2} \int_{\mathbf{T}} \frac{1+z \zeta}{1-z \zeta} d \mu(\zeta) .
$$

Thus $\psi_{\mu}(z)$ is analytic for $|z|<1$ and, for such $z$,

$$
\Re \psi_{\mu}(z)=-\frac{1}{2}+\frac{1}{2} \int_{\mathbf{T}} \frac{1-|z|^{2}}{|1-z \zeta|^{2}} d \mu(\zeta) \geq-\frac{1}{2}
$$


We conclude from the theory of Nevanlinna-Pick interpolation that given $a_{1}, a_{2}, \ldots, a_{n}$ with $\left|a_{j}\right|<1, j=1,2, \ldots, n$, the matrix

$$
\left[\frac{\psi_{\mu}\left(a_{j}\right)+\frac{1}{2}+\overline{\psi_{\mu}\left(a_{k}\right)+\frac{1}{2}}}{1-a_{j} \bar{a}_{k}}\right]_{j, k=1}^{n}=\left[\frac{\psi_{\mu}\left(a_{j}\right)+\overline{\psi_{\mu}\left(a_{k}\right)}+1}{1-a_{j} \bar{a}_{k}}\right]_{j, k=1}^{n}
$$

is nonnegative definite. If $z_{1}, z_{2}, \ldots, z_{n}$ are sufficiently close to zero, we can take $a_{j}=\chi_{\mu}\left(z_{j}\right), j=1,2, \ldots, n$, and we deduce immediately the necessity of condition (2).

Conversely, let $S$ satisfy conditions (1) and (2). Define

$$
\chi(z)=\frac{z}{z+1} S(z)
$$

and note that $\chi(0)=0$ and $\chi^{\prime}(0)=S(0) \neq 0$ so that $\chi$ is locally invertible at $z=0$. Denote by $\psi(z)$ the local inverse of $\chi$, defined in a neighborhood of $z=0$. Condition (2) implies that for $a_{1}, a_{2}, \ldots, a_{n}$ in a neighborhood of zero, the matrix

$$
\left[\frac{u\left(a_{j}\right)+\overline{u\left(a_{k}\right)}}{1-a_{j} \bar{a}_{k}}\right]_{j, k=1}^{n}
$$

is nonnegative definite, where $u(z)=\psi(z)+1 / 2$. Using again the theory of the Nevanlinna-Pick interpolation problem we deduce that $u$ extends to an analytic function such that $\Re u(z) \geq 0$ for all $z$ in the unit disc. Indeed, let $a_{n}$ be a sequence of distinct points converging to zero. There exist functions $u_{n}$ analytic in the unit disc and with positive real part such that $u_{n}\left(a_{j}\right)=u\left(a_{j}\right)$ for $j=1,2, \ldots, n$. Dropping, if necessary, to a subsequence we may assume that the $u_{n}$ converge uniformly on compact subsets of the unit disc to a function $v$. We have $\Re v(z) \geq 0$ for $|z|<1$ and $v\left(a_{j}\right)=u\left(a_{j}\right)$ for all $j$, which implies immediately that $v$ is an analytic continuation of $u$ to the unit disc. Note that $v(0)=u(0)=1 / 2$, and a theorem of Herglotz implies that we can write

$$
v(z)=\frac{1}{2} \int_{\mathrm{T}} \frac{1+z \zeta}{1-z \zeta} d \mu(\zeta), \quad|z|<1,
$$

for some measure $\mu \in \mathscr{M}_{\mathbf{T}}$. We conclude at once that $\psi=\psi_{\mu}$, and hence $\mu \in \mathscr{M}_{*}$ because $\psi$ is locally invertible at zero. Thus we have $\mu \in \mathscr{M}_{\mathbf{T}} \cap \mathscr{M}_{*}$ and $S=S_{\mu}$, as desired.

In order to verify the last statement of the proposition, assume that $\left|\chi_{\mu}(z)\right|<1$ for $|z|<\varepsilon$. Then $\psi_{\mu}\left(\chi_{\mu}(z)\right)$ makes sense for $|z|<\varepsilon$, and since $\psi_{\mu}\left(\chi_{\mu}(z)\right)=z$ for $z$ close to zero, we must have $\psi_{\mu}\left(\chi_{\mu}(z)\right)=z$ 
for $|z|<\varepsilon$. The claim now follows from the first part of the proof since we can take $a_{j}=\chi_{\mu}\left(z_{j}\right)$ if $\left|z_{j}\right|<\varepsilon$ for $j=1,2, \ldots, n$.

We note for further reference the following extreme case of Proposition 2.2.

2.3. Lemma. Assume that $\mu \in \mathscr{M}_{\mathrm{T}} \cap \mathscr{M}_{*}$ is a measure satisfying the condition $\left|S_{\mu}(0)\right|=1$. Then $\mu=\delta_{\alpha}$ for some $\alpha \in \mathbf{T}$.

Proof. Observe that $\left|\psi_{\mu}^{\prime}(0)\right|=1$, and choose $\alpha \in \mathbf{T}$ such that $\bar{\alpha} \psi_{\mu}^{\prime}(0)=1$. Then

$$
\int_{\mathbf{T}} \Re(1-\bar{\alpha} \zeta) d \mu(\zeta)=\Re \int_{\mathbf{T}}(1-\bar{\alpha} \zeta) d \mu(\zeta)=0,
$$

which implies that the nonnegative function $\Re(1-\bar{\alpha} \zeta)$ must vanish almost everywhere with respect to $\mu$. We conclude that $\mu=\delta_{\alpha}$.

2.4. Corollary. Let $\mu_{i} \in \mathscr{M}_{\mathrm{T}} \cap \mathscr{M}_{*}, i=1,2, \ldots$, be such that the functions $S_{\mu_{i}}(z)$ converge uniformly in some neighborhood of $z=0$ to a function $S(z)$. Then there exists $\mu \in \mathscr{M}_{\mathrm{T}} \cap \mathscr{M}_{*}$ such that $S=S_{\mu}$.

Proof. Clearly $|S(0)| \geq 1$, and therefore it suffices to show that $S$ satisfies condition (2) of Proposition 2.2. Indeed, denote $\chi(z)=$ $z S(z) /(z+1)$, and choose $\varepsilon>0$ so small that $|\chi(z)|<1 / 2$ and $\left|\chi(z)-\chi_{\mu_{i}}(z)\right|<1 / 2$ for $|z|<\varepsilon$ and $i$ sufficiently large. Then clearly $\left|\chi_{\mu_{i}}(z)\right|<1$ for $|z|<\varepsilon$, and therefore the matrix

$$
\left[\frac{z_{j}+\bar{z}_{k}+1}{1-\chi_{\mu_{i}}\left(z_{j}\right) \overline{\chi_{\mu_{i}}\left(z_{k}\right)}}\right]_{j, k=1}^{n}
$$

is nonnegative definite if $\left|z_{j}\right|<\varepsilon, j=1,2, \ldots, n$. Thus the matrix

$$
\left[\frac{z_{j}+\bar{z}_{k}+1}{1-\chi\left(z_{j}\right) \overline{\chi\left(z_{k}\right)}}\right]_{j, k=1}^{n}=\lim _{i \rightarrow \infty}\left[\frac{z_{j}+\bar{z}_{k}+1}{1-\chi_{\mu_{i}}\left(z_{j}\right) \overline{\chi_{\mu_{i}}\left(z_{k}\right)}}\right]_{j, k=1}^{n}
$$

is also nonnegative definite.

It is not difficult to show that actually the weak* convergence of a sequence of measures in $\mathscr{M}_{\mathrm{T}} \cap \mathscr{M}_{*}$ is equivalent to the uniform convergence of the corresponding functions $S$ in some neighborhood of zero. 
2.5. Proposition. Consider a measure $\mu \in \mathscr{M}_{\mathrm{T}} \cap \mathscr{M}_{*}$, and a sequence $\mu_{i} \in \mathscr{M}_{\mathrm{T}}, i=1,2, \ldots$ If $\mu_{i}$ converge to $\mu$ in the weak ${ }^{*}$ topology, then $\mu_{i} \in \mathscr{M}_{\mathrm{T}} \cap \mathscr{M}_{*}$ eventually, and the functions $S_{\mu_{i}}$ converge uniformly to $S_{\mu}$ in some neighborhood of zero. Conversely, if $\mu_{i} \in \mathscr{M}_{\mathrm{T}} \cap \mathscr{M}_{*}$ and $S_{\mu_{i}}$ converge uniformly to $S_{\mu}$ in some neighborhood of zero, then the measures $\mu_{i}$ converge to $\mu$ in the weak* topology.

Proof. Assume first that $\mu_{i}$ converge to $\mu$ in the weak* topology. Clearly then the functions $\psi_{\mu_{i}}$ converge uniformly on the compact subsets of the unit disc to $\psi_{\mu}$, and therefore we can find an integer $n$, and positive numbers $\varepsilon, \delta$ such that, for $i \geq n$, the function $\psi_{\mu_{l}} \mid\{z:|z|<\varepsilon\}$ is one-to-one, and onto some neighborhood of $\{z$ : $|z|<\delta\}$. Clearly, for $i \geq n, \mu_{i}$ belongs to $\mathscr{M}_{\mathbf{T}} \cap \mathscr{M}_{*}$, and the functions $\chi_{\mu_{t}(z)}$ and $S_{\mu_{t}(z)}$ are defined for $|z|<\delta$.

Observe now that there exist polynomials $P_{n}$ in $n$ variables such that, given two inverse (formal) power series $\sum_{n=1}^{\infty} a_{n} z^{n}$ and $\sum_{n=1}^{\infty} b_{n} z^{n}$, we have

$$
a_{n}=b_{1}^{-n !} P_{n}\left(b_{1}, b_{2}, \ldots, b_{n}\right), \quad n=1,2, \ldots
$$

It follows immediately that the coefficients of the Taylor series of $\chi_{\mu}$ converge to the corresponding coefficients of $\chi_{\mu}$, and since these functions are bounded by $\varepsilon$ in $\{z:|z|<\delta\}$, we conclude that the functions $\chi_{\mu_{i}}$ converge uniformly to $\chi_{\mu}$ on the compact subsets of this disc. Thus $S_{\mu}$ also converge uniformly to $S_{\mu}$ in some neighborhood of zero.

Conversely, assume now that $\mu_{i} \in \mathscr{M}_{\mathrm{T}} \cap \mathscr{M}_{*}$, and $S_{\mu_{i}}$ converge uniformly to $S_{\mu}$ in some neighborhood of zero. The first part of the argument and Lemma 2.1 show that the only limit point of the sequence $\left\{\mu_{i}\right\}$ in the weak* topology is the measure $\mu$. The desired conclission follows now from the weak*-compactness of $\mathscr{M}_{\mathbf{T}}$.

One can prove analogous results for the function $\Sigma_{\mu}$. Since the proofs are very similar, we only give here the statements; the basic additional observation needed is that $\left|\psi_{\mu}^{\sim}(z)\right| \leq 1$ for $z$ in the unit disc.

2.6. Proposition. Let $\Sigma(z)$ be a convergent power series. Then $\Sigma=\Sigma_{\mu}$ for some $\mu \in \mathscr{M}_{\mathrm{T}} \cap \mathscr{M}_{*}$ if and only if the following conditions are satisfied:

(1) $|\Sigma(0)| \geq 1$; 
(2) there exists $\varepsilon>0$ such that, given a natural number $n$ and points $z_{1}, z_{2}, \ldots, z_{n} \in \mathbf{C}$ with $\left|z_{j}\right|<\varepsilon, j=1,2, \ldots, n$, the matrix

$$
\left[\frac{1-z_{j} \overline{z_{k}}}{1-z_{j} \Sigma\left(z_{j}\right) \overline{z_{k} \Sigma\left(z_{k}\right)}}\right]_{j, k=1}^{n}
$$

is nonnegative definite.

If $\Sigma=\Sigma_{\mu}$ then the number $\varepsilon$ can be chosen to be the greatest with the property that $\left|\chi_{\mu}^{\sim}(z)\right|<1$ for $|z|<\varepsilon$.

2.7. Lemma. Assume that $\mu \in \mathscr{M}_{\mathrm{T}} \cap \mathscr{M}_{*}$ is a measure satisfying the condition $\left|\Sigma_{\mu}(0)\right|=1$. Then $\mu=\delta_{\alpha}$ for some $\alpha \in \mathbf{T}$.

2.8. Corollary. Let $\mu_{i} \in \mathscr{M}_{\mathbf{T}} \cap \mathscr{M}_{*}, i=1,2, \ldots$, be such that the functions $\Sigma_{\mu_{i}}(z)$ converge uniformly in some neighborhood of $z=0$ to a function $\Sigma(z)$. Then there exists $\mu \in \mathscr{M}_{\mathbf{T}} \cap \mathscr{M}_{*}$ such that $\Sigma=\Sigma_{\mu}$.

2.9. Proposition. Consider a measure $\mu \in \mathscr{M}_{\mathrm{T}} \cap \mathscr{M}_{*}$, and a sequence $\mu_{i} \in \mathscr{M}_{\mathrm{T}}, i=1,2, \ldots$. If $\mu_{i}$ converge to $\mu$ in the weak* topology, then $\mu_{i} \in \mathscr{M}_{\mathrm{T}} \cap \mathscr{M}_{*}$ eventually, and the functions $\Sigma_{\mu_{i}}$ converge uniformly to $\Sigma_{\mu}$ in some neighborhood of zero. Conversely, if $\mu_{i} \in \mathscr{M}_{\mathrm{T}} \cap \mathscr{M}_{*}$ and $\Sigma_{\mu_{i}}$ converge uniformly to $\Sigma_{\mu}$ in some neighborhood of zero, then the measures $\mu_{i}$ converge to $\mu$ in the weak* topology.

3. The function $S_{\mu}$ for $\mu \in \mathscr{M}_{\mathbf{R}_{+}}$. The analogues of the results proved in the preceding section are somewhat more involved for measures in $\mathscr{M}_{\mathbf{R}_{+}}$.

3.1. Proposition. Let $S(z)$ be a convergent power series. Then $S=S_{\mu}$ for some probability measure $\mu \in \mathscr{M}_{\mathbf{R}_{+}}$if and only if the following conditions are satisfied:

(1) $S$ can be continued analytically to a neighborhood of the interval $[-1,0]$;

(2) $S^{\prime}(z) \leq 0$ for $z \in[-1,0]$

(3) $S(z)>0$ for $z \in[-1,0]$;

(4) $S(\bar{z})=\overline{S(z)}$;

(5) there is $\varepsilon>0$ such that, given a natural number $n$, and points $z_{1}, z_{2}, \ldots, z_{n} \in \mathbf{C}$ with $\left|z_{j}\right|<\varepsilon$ and $\Im z_{j}>0, j=1,2, \ldots, n$, the 
matrix

is nonnegative definite.

$$
\left[\frac{z_{j}-\bar{z}_{k}}{\frac{z_{j} S\left(z_{j}\right)}{1+z_{j}}-\frac{\bar{z}_{k} S\left(\bar{z}_{k}\right)}{1+\bar{z}_{k}}}\right]_{j, k=1}^{n}
$$

If $S=S_{\mu}$ then the number $\varepsilon$ can be chosen to be the greatest with the property that $\Im \chi_{\mu}(z)>0$ for $|z|<\varepsilon, \Im z>0$.

Proof. Start first with a measure $\mu \in \mathscr{M}_{\mathbf{R}_{+}}$. Observe that $\psi_{\mu}(z)$ is defined for $z \in \mathbf{C} \backslash[\eta, 1 / \eta]$, where $\eta$ is chosen so that $t^{-1} \in[\eta, 1 / \eta]$ for every $t \in \operatorname{supp}(\mu)$. In fact $\psi_{\mu}$ is also analytic at $\infty$ because

$$
\begin{aligned}
\psi_{\mu}\left(\frac{1}{z}\right) & =\int_{0}^{\infty} \frac{t}{z-t} d \mu(t)=-\int_{0}^{\infty} \frac{1}{1-\frac{z}{t}} d \mu(t) \\
& =-\sum_{n=0}^{\infty} z^{n} \int_{0}^{\infty} t^{-n} d \mu(t) .
\end{aligned}
$$

Hence $\psi_{\mu}(\infty)=-1$ and $\psi_{\mu}$ is locally invertible at $\infty$. Furthermore, the derivative

$$
\psi_{\mu}^{\prime}(z)=\int_{0}^{\infty} \frac{t}{(1-z t)^{2}} d \mu(t)
$$

is positive if $z \leq 0$. We deduce from these facts that the inverse function $\chi_{\mu}$ to $\psi_{\mu}$ has a meromorphic continuation to a neighborhood of $[-1,0]$, with a simple pole at $z=-1$, a simple zero at $z=0, \chi_{\mu}(z)<0$ and $\chi_{\mu}^{\prime}(z)>0$ for $z \in(-1,0]$. We see therefore that $S_{\mu}(z)=(z+1) \chi_{\mu}(z) / z$ is analytic and nonzero in a neighborhood of $[-1,0]$, and $S_{\mu}(z)>0$ for $z \in[-1,0]$, while $S_{\mu}(z)+$ $z(z+1) S_{\mu}^{\prime}(z)=(z+1)^{2} \psi_{\mu}(z)^{\prime}>0$ if $z \in(-1,0)$. Since $S_{\mu}(z)^{n}$ has the form $S_{\nu}(z)$ for some $\nu \in \mathscr{M}_{\mathbf{R}_{+}}$, we also have $S_{\mu}(z)^{n}+$ $z(z+1) n S_{\mu}^{\prime}(z) S_{\mu}(z)^{n-1}>0$ for $z \in(-1,0)$ and $n=1,2, \ldots$ Letting $n$ tend to $\infty$ we conclude that $S_{\mu}^{\prime}(z) \leq 0$ for $z \in(-1,0)$. Thus $S_{\mu}$ satisfies conditions (1)-(4) in the statement. In order to verify (5) we calculate the imaginary part of $\psi_{\mu}$ :

$$
\Im \psi_{\mu}(z)=\int_{0}^{\infty} \Im \frac{z t}{1-z t} d \mu(t)=\int_{0}^{\infty} \frac{t \Im z}{|1-z t|^{2}} d \mu(t)
$$

and we see that $\Im \psi_{\mu}(z)>0$ for $\Im z>0$. The Nevanlinna-Pick theory for the upper half-plane shows that for any finite number of points $a_{1}, a_{2}, \ldots, a_{n}$ with positive imaginary parts, the matrix

$$
\left[\frac{\psi_{\mu}\left(a_{j}\right)-\overline{\psi_{\mu}\left(a_{k}\right)}}{a_{j}-\bar{a}_{k}}\right]_{j, k=1}^{n}
$$


is nonnegative definite. Choose $\varepsilon$ small enough that $\Im \chi_{\mu}(z)>0$ for $|z|<\varepsilon, \Im z>0$. In the above matrix we can replace $a_{1}, a_{2}, \ldots, a_{n}$ by $\chi_{\mu}\left(z_{1}\right), \chi_{\mu}\left(z_{2}\right), \ldots, \chi_{\mu}\left(z_{n}\right)$ with $\Im z_{j}>0$ and $\left|z_{j}\right|<\varepsilon$ for $j=$ $1,2, \ldots, n$, and we see in this case that the matrix

$$
\left[\frac{z_{j}-\bar{z}_{k}}{\chi_{\mu}\left(z_{j}\right)-\overline{\chi_{\mu}\left(z_{k}\right)}}\right]_{j, k=1}^{n}
$$

is nonnegative definite. This last condition is equivalent to (5).

Conversely, assume now that $S$ is a function satisfying conditions (1)-(5). Define $\chi(z)=z S(z) /(z+1)$, and note that $\chi$ is meromorphic in a neighborhood of $[-1,0]$, it has a simple pole at $z=-1$, $\chi(z)<0$ for $z \in(-1,0]$, and $\chi^{\prime}(z)>0$ for $z \in(-1,0]$. Therefore $\chi$ is locally invertible at every point of $[-1,0]$, and hence it has an inverse function $\psi$ defined in a neighborhood of $(-\infty, 0] \cup\{\infty\}$ such that $\psi(0)=0$ and $\psi(\infty)=-1$. Moreover, condition (v) imples that the matrix

$$
\left[\frac{\psi_{\mu}\left(a_{j}\right)-\overline{\psi_{\mu}\left(a_{k}\right)}}{a_{j}-\bar{a}_{k}}\right]_{j, k=1}^{n}
$$

is nonnegative definite if $a_{1}, a_{2}, \ldots, a_{n}$ have positive imaginary parts and are sufficiently small. Using again the theory of the NevanlinnaPick interpolation we see (as in the proof of Proposition 2.2) that $\psi$ has an analytic continuation in the upper half-plane. Thus in fact $\psi$ extends to the complement of some interval of the form $[\eta, 1 / \eta]$, and $\Im \psi(z)>0$ if $\Im z>0$. The function $u(z)=\psi(1 / z)$ then has negative imaginary part in the upper half-plane, and $u(\infty)=0$, so that there exists a positive measure $\nu$ with $\operatorname{supp}(\nu) \subset[\eta, 1 / \eta]$ satisfying

$$
u(z)=\int_{0}^{\infty} \frac{1}{z-t} d \nu(t), \quad z \notin[\eta, 1 / \eta] .
$$

We define now a measure $\mu$ by $d \mu(t)=t^{-1} d \nu(t)$, and we see that

$$
\psi(z)=u(1 / z)=\int_{0}^{\infty} \frac{1}{\frac{1}{z}-t} d \nu(t)=\int_{0}^{\infty} \frac{z t}{1-z t} d \mu(t)
$$

for $z \notin[\eta, 1 / \eta]$. Moreover, we have $\int_{0}^{\infty} d \mu(t)=-\psi(\infty)=1$, so that $\mu \in \mathscr{M}_{\mathbf{R}_{+}}$and $S=S_{\mu}$.

To prove the last part of the statement assume that $S=S_{\mu}$, and let $\varepsilon$ be such that $\Im \chi_{\mu}(z)>0$ if $\Im z>0$ and $|z|<\varepsilon$. As in the proof of Proposition 2.2 we see that $\psi(\chi(z))=z$ for $\Im z>0,|z|<\varepsilon$, and thus condition (5) is satisfied in the required range. 
3.2. Corollary. Let $\mu_{i} \in \mathscr{M}_{\mathbf{R}_{+}}, i=1,2, \ldots$, be such that the functions $S_{\mu}$ converge uniformly in some neighborhood of $z=0$ to a function $S(z)$. If $S$ satisfies conditions (1)-(4) of Proposition 3.1, then there exists $\mu \in \mathscr{M}_{\mathbf{R}_{+}}$such that $S=S_{\mu}$.

Proof. We must show that $S$ also satisfies condition (5) of Proposition 3.1. Since $S$ satisfies conditions (1)-(4), we deduce that the function $\chi(z)=z S(z) /(z+1)$ is real for small, real $z$, and $\chi^{\prime}(0)>0$. Choose $\varepsilon>0$ small enough so that $\chi(z)$ is one-to-one and $\chi_{\mu_{t}}(z) \rightarrow$ $\chi(z)$ uniformly for $|z| \leq \varepsilon$. Then $\chi_{\mu_{i}}(z)$ must also be one-to-one for $|z| \leq \varepsilon$ provided that $i$ is large enough. We claim that for such $i$ we have $\Im \chi_{\mu_{i}}(z)>0$ if $\Im z>0$ and $|z|<\varepsilon$. Indeed, we know that $\Im \chi_{\mu_{t}}(z)>0$ if $\Im z>0$ and $z$ is sufficiently close to zero. If there were $z$ with $|z|<\varepsilon$ such that $\Im z>0$ and $\Im \chi_{\mu_{t}}(z) \leq 0$, we would deduce by connectedness the existence of such a $z$ with $\chi_{\mu_{i}}(z) \in \mathbf{R}$. But then $\chi_{\mu_{\imath}}(\bar{z})=\overline{\chi_{\mu_{\imath}}(z)}=\chi_{\mu_{\imath}}(z)$, thus contradicting the injectivity of $\chi_{\mu_{t}}(z)$ for $|z|<\varepsilon$.

We are now ready to verify condition (5) as in the proof of Corollary 2.4. Let $z_{1}, z_{2}, \ldots, z_{n}$ be such that $\left|z_{j}\right|<\varepsilon$ and $\Im z_{j}>0$ for $j=1,2, \ldots, n$. By virtue of the last statement in Proposition 3.1, the matrix

$$
\left[\frac{z_{j}-\overline{z_{k}}}{\chi_{\mu_{i}}\left(z_{j}\right)-\overline{\chi_{\mu_{i}}\left(z_{k}\right)}}\right]_{j, k=1}^{n}
$$

is nonnegative definite. We deduce that

$$
\left[\frac{z_{j}-\overline{z_{k}}}{\chi\left(z_{j}\right)-\overline{\chi\left(z_{k}\right)}}\right]_{j, k=1}^{n}=\lim _{i \rightarrow \infty}\left[\frac{z_{j}-\overline{z_{k}}}{\chi_{\mu_{\imath}}\left(z_{j}\right)-\overline{\chi_{\mu_{t}}\left(z_{k}\right)}}\right]_{j, k=1}^{n}
$$

is also nonnegative definite.

In order to prove the analogue of Proposition 2.5, we remark that weak*-convergence of probability measures supported in a fixed compact interval of the real line makes sense, and it is equivalent to the convergence of all the moments of the given measures. It will be convenient to denote by $[-\infty, 0]$ the subset $(-\infty, 0] \cup\{\infty\}$ of the extended complex plane.

3.3. Proposition. Given a compact interval $[a, b] \subset \mathbf{R}_{+}$, there exist an open neighborhood $U$ of $[-\infty, 0]$ and an open neighborhood $V$ of $[-1,0]$ with the following property: for every measure $\mu \in \mathscr{M}_{\mathbf{R}_{+}}$with support in $[a, b]$, the function $\psi_{\mu}$ is invertible on $U$, and $\psi_{\mu}(U)^{+} \supset V$. Moreover, a sequence $\mu_{i} \in \mathscr{M}_{\mathbf{R}_{+}}, i=1,2, \ldots$, of measures supported 
in $[a, b]$ converges in the weak $k^{*}$ topology to a measure $\mu$, if and only if the functions $S_{\mu_{i}}$ converge uniformly to $S_{\mu}$ on the compact subsets of $V$.

Proof. For every positive number $M$, the function $t /(1-z t)^{2}$ is positive and bounded away from zero for $z \in[-M, 0]$ and $t \in$ $[a, b]$. It follows that there exists a convex neighborhood $W_{M}$ of $[-M, 0]$, and a positive number $\varepsilon$, such that $\Re\left(t /(1-z t)^{2}\right) \geq \varepsilon$ for all $z \in W_{M}$ and $t \in[a, b]$. We conclude that $\left|\psi_{\mu}\left(z_{1}\right)-\psi_{\mu}\left(z_{2}\right)\right| \geq$ $\varepsilon\left|z_{1}-z_{2}\right|$ if $z_{1}, z_{2} \in W_{M}$ and $\mu$ is supported in $[a, b]$. In an analogous manner, we can choose $\delta, \eta>0$ such that $\mid \psi_{\mu}\left(1 / z_{1}\right)-$ $\psi_{\mu}\left(1 / z_{2}\right)|\geq \eta| 1 / z_{1}-1 / z_{2} \mid$ if $\left|z_{1}\right|,\left|z_{2}\right|<\delta$ and $\mu$ is supported in $[a, b]$. Clearly we can choose $M$ and $\delta$ such that $M>1 / \delta$, and the sets $\left\{\psi_{\mu}(z):|z|>M\right\}$ and $\left\{\psi_{\mu}(z): z \in W_{M},|z|<1 / \delta\right\}$ are disjoint. With such a choice we set $U=W_{M} \cup\{z:|z|>1 / \delta\}$, and we see that $\psi_{\mu}$ is one-to-one on $U$ if $\mu$ is supported in $[a, b]$. It is easy to see now that the first assertion of the proposition is verified if we take $V=\{z: \operatorname{dist}(z,[-1,0])<\alpha\}$, provided that $\alpha \leq \min (\varepsilon, \eta)$.

Since the collection of probability measures supported in $[a, b]$ is compact in the weak* topology, while the corresponding family of functions $S_{\mu}$ is a normal family of analytic functions on $V$, it suffices to show that the weak* convergence of the measures $\mu_{i}$ to $\mu$ implies the uniform convergence of $S_{\mu_{i}}$ to $S_{\mu}$ in some neighborhood of zero. This last fact is proved as in Proposition 2.5.

3.4. Proposition. Let $\mu_{i} \in \mathscr{M}_{\mathbf{R}_{+}}, i=1,2, \ldots$, be a sequence such that the functions $S_{\mu_{i}}$ converge uniformly in a neighborhood of $\{0\} \cup\{-1\}$ to a function $S$ such that $S(0) \neq 0$ and $S(-1) \neq 0$. Then the measures $\mu_{i}$ are supported in some compact interval, and they converge in the weak ${ }^{*}$ topology to some measure $\mu$.

Proof. It follows immediately from Proposition 3.3 that it suffices to prove that the supports of the measures $\mu_{i}$ are bounded away from 0 and $\infty$. Observe that the derivatives $\chi_{\mu_{i}}^{\prime}(z)$ converge uniformly in a neighborhood of zero to a function which does not vanish. We conclude that there exist $\varepsilon, \delta>0$ such that $\chi_{\mu_{i}}^{\prime}(z)>\delta$ for $z \in[-\varepsilon, \varepsilon]$ and all $i$. Therefore $\chi_{\mu_{i}}(\varepsilon)=\int_{0}^{\varepsilon} \chi_{\mu_{i}}^{\prime}(z) d z>\varepsilon \delta$ for all $i$. We conclude that the inverse $\psi_{\mu_{i}}$ of $\chi_{\mu_{i}}$ extends analytically to the segment $[0, \varepsilon \delta]$, and hence the support of $\mu_{i}$ lies to the left of $1 / \varepsilon \delta$. In an analogous manner, there exist $\varepsilon^{\prime}, \delta^{\prime}>0$ such that $d\left(1 / \chi_{\mu_{i}}(z)\right) / d z<\delta^{\prime}$ for $z \in\left[-1-\varepsilon^{\prime},-1+\varepsilon^{\prime}\right]$, and this implies 
that $\psi_{\mu_{1}}(1 / z)$ can be continued analytically to $\left[0, \varepsilon^{\prime} \delta^{\prime}\right]$. This in turn implies that the support of $\mu_{i}$ lies to the right of $\varepsilon^{\prime} \delta^{\prime}$.

We record for future reference the corresponding results for the function $\Sigma_{\mu}$.

3.5. Proposition. Let $\Sigma(z)$ be a convergent power series. Then $\Sigma=\Sigma_{\mu}$ for some probability measure $\mu \in \mathscr{M}_{\mathbf{R}_{+}}$if and only if the following conditions are satisfied:

(1) $\Sigma$ can be continued analytically to a neighborhood of the interval $[-\infty, 0]$

(2) $\Sigma^{\prime}(z) \leq 0$ for $z \in(-\infty, 0]$;

(3) $\Sigma(z)>0$ for $z \in[-\infty, 0]$;

(4) $\Sigma(\bar{z})=\overline{\Sigma(z)}$;

(5) there is $\varepsilon>0$ such that, given a natural number $n$, and points $z_{1}, z_{2}, \ldots, z_{n} \in \mathbf{C}$ with $\left|z_{j}\right|<\varepsilon$ and $\Im z_{j}>0, j=1,2, \ldots, n$, the matrix

$$
\left[\frac{z_{j}-\bar{z}_{k}}{z_{j} \Sigma\left(z_{j}\right)-\bar{z}_{k} \Sigma\left(\bar{z}_{k}\right)}\right]_{j, k=1}^{n}
$$

is nonnegative definite.

If $\Sigma=\Sigma_{\mu}$ then the number $\varepsilon$ can be chosen to be the greatest with the property that $\Im \chi_{\mu}(z)>0$ for $|z|<\varepsilon, \Im z>0$.

3.6. Corollary. Let $\mu_{i} \in \mathscr{M}_{\mathbf{R}_{+}}, i=1,2, \ldots$, be such that the functions $\Sigma_{\mu_{i}}$ converge uniformly in some neighborhood of $z=0$ to a function $\Sigma(z)$. If $\Sigma$ satisfies conditions (1)-(4) of Proposition 3.5 then there exists $\mu \in \mathscr{M}_{\mathbf{R}_{+}}$such that $\Sigma=\Sigma_{\mu}$.

3.7. Proposition. Given a compact interval $[a, b] \subset \mathbf{R}_{+}$, there exist open neighborhoods $U$ and $V$ of $[-\infty, 0]$ with the following property: for every measure $\mu \in \mathscr{M}_{\mathbf{R}_{+}}$with support in $[a, b]$, the function $\psi_{\mu}$ is invertible on $U$, and $\psi_{\mu}(U) \supset V$. Moreover, a sequence $\mu_{i} \in \mathscr{M}_{\mathbf{R}_{+}}, i=1,2, \ldots$, of measures supported in $[a, b]$ converges in the weak* topology to a measure $\mu$, if and only if the functions $\Sigma_{\mu_{i}}$ converge uniformly to $\Sigma_{\mu}$ on the compact subsets of $V$.

3.8. Proposition. Let $\mu_{i} \in \mathscr{M}_{\mathbf{R}_{+}}, i=1,2, \ldots$, be a sequence such that the functions $\Sigma_{\mu}$ converge uniformly in a neighborhood of $\{0\} \cup\{\infty\}$ to a function $\Sigma$ such that $\Sigma(0) \neq 0$ and $\Sigma(\infty) \neq 0$. Then the measures $\mu_{i}$ are supported in some compact interval, and they converge in the weak ${ }^{*}$ topology to some measure $\mu$. 
4. Some examples. All the basic examples of $\otimes$-infinitely divisible probability measures will come from limits of multiplicative free products of atomic measures. In fact, our analogues of the Gaussian and Poisson distributions are completely analogous with de Moivre's theorem (for the normal law) and the usual limiting process for the Poisson law. The following calculation is useful here.

4.1. Lemma. (1) Let $a, b \in \mathbf{C}, a b \neq 0$, and $\alpha, \beta \in(0,1)$ be such that $\alpha+\beta=1$ and $\alpha a+\beta b \neq 0$. Then $\mu=\alpha \delta_{a}+\beta \delta_{b} \in \mathscr{M}_{*}$, and

$$
\begin{aligned}
S_{\mu}(z) & =\frac{\alpha a+\beta b+a z+b z-\sqrt{(\alpha a+\beta b+a z+b z)^{2}-4 a b z(z+1)}}{2 a b z} \\
& =\frac{2(z+1)}{\alpha a+\beta b+a z+b z+\sqrt{(\alpha a+\beta b+a z+b z)^{2}-4 a b z(z+1)}},
\end{aligned}
$$

for $z$ close to zero, where the branch of the square root is determined such that the numerator of the first expression above vanishes for $z=0$.

(2) If $a \neq 0, b=0, \alpha \in(0,1]$, and $\beta=1-\alpha$, then $\mu=$ $\alpha \delta_{a}+\beta \delta_{b} \in \mathscr{M}_{*}$, and

$$
S_{\mu}(z)=a^{-1} \frac{z+1}{z+\alpha}, \quad z \neq-\alpha .
$$

(3) If $a \neq 0,|a|<1$, and $P_{a} \in \mathscr{M}_{\mathrm{T}}$ is defined as in $\S 2$, then $P_{a} \in \mathscr{M}_{*}$ and $S_{P_{a}}(z)=a^{-1}$.

Proof. (1) We have $\mu \in \mathscr{M}_{*}$ because $\int_{\mathbf{C}} d \mu=\alpha a+\beta b \neq 0$. We now calculate

$$
\psi_{\mu}(z)=\alpha \frac{a z}{1-a z}+\beta \frac{b z}{1-b z} .
$$

To calculate $\chi_{\mu}(z)$ we use the equation $\psi_{\mu}\left(\chi_{\mu}(z)\right)=z$ which becomes, after obvious modifications,

$$
a b(z+1) \chi_{\mu}(z)^{2}-(\alpha a+\beta b+a z+b z) \chi_{\mu}(z)+z=0 .
$$

The formula for $S_{\mu}(z)=(z+1) \chi_{\mu}(z) / z$ is simply obtained by solving this quadratic equation, keeping in mind the fact that $\chi_{\mu}(0)=0$.

(2) This is even easier since $\psi_{\mu}(z)=\alpha a z /(1-a z)$.

(3) We saw earlier that $S_{P_{a}}=S_{\delta_{a}}$ so that (3) follows readily from (2) if we take $\alpha=1$. 
4.2. Corollary. We have $P_{a} \otimes P_{b}=P_{a b}$ for $|a|<1,|b|<1$.

Proof. If $a b \neq 0$ then $S_{P_{a}} S_{P_{b}}=S_{P_{a b}}$ and the corollary follows from Lemma 1. If $a b=0$ and $\mu=P_{a} \otimes P_{b}$, then we have $\int_{\mathbf{T}} z^{n} d \mu(z)=$ $0=\int_{\mathbf{T}} z^{n} d P_{0}$, so that $\mu=P_{0}$ by Lemma 2.1 .

5. A result about free convolution semigroups. In this section we consider a $\otimes$-semigroup $\left\{\mu_{t}: t \geq 0\right\}$ (that is, $\left.\mu_{t+s}=\mu_{t} \otimes \mu_{s}\right)$ such that $\Sigma_{\mu_{t}}(z)=\exp (t u(z))$ in some neighborhood (depending on $t$ ) of zero, where $u(z)$ is some convergent power series. If we denote

$$
\psi(t, z)=\psi_{\mu_{t}}^{\sim}(z), \quad \chi(t, z)=\chi_{\mu_{t}}^{\sim}(z), \quad t \geq 0,
$$

then we have $\chi(t, z)=z \exp (t u(z))$, and $\psi(t, \chi(t, z))=z$ for $t \geq$ 0 . It is clear that the function $\psi$ can be extended analytically to some neighborhood of $\mathbf{C} \times\{0\}$, such that the identity

$$
\psi(t, z \exp (t u(z)))=z
$$

holds. Differentiation in $t$ of this identity yields the following partial differential equation which $\psi$ must satisfy:

$$
\frac{\partial \psi}{\partial t}(t, z \exp (t u(z)))+z u(z) \exp (t u(z)) \frac{\partial \psi}{\partial z}(t, z \exp (t u(z)))=0 .
$$

We note that this equation was first proved in [7] for the functions $S_{\mu}$.

5.1. Proposition. For $z$ in some neighborhood of zero we have

$$
u(z)=\lim _{\varepsilon \rightarrow 0} \frac{1}{\varepsilon} \int_{\mathbf{C}} \frac{(1-\tau)(1-z)}{1-\tau z} d \mu_{\varepsilon}(\tau) .
$$

Proof. Setting $t=0$ in the partial differential equation satisfied by $\psi$ we get

$$
\frac{\partial \psi}{\partial t}(0, z)+z u(z) \frac{\partial \psi}{\partial z}(0, z)=0
$$

or, equivalently since $\psi(0, z)=z$,

$$
u(z)=-\frac{1}{z} \frac{\partial \psi}{\partial t}(0, z)=-\left.\frac{1}{z} \frac{\partial \psi_{\mu_{t}}^{\tilde{t}}}{\partial t}(z)\right|_{t=0} .
$$

Using the relation $\psi_{\mu}^{\tilde{\mu}}=\psi_{\mu} /\left(1+\psi_{\mu}\right)$ and the fact that $\psi_{\mu_{0}}(z)=$ $z /(1-z)$ we deduce that 


$$
\begin{aligned}
u(z) & =-\left.\frac{1}{z} \frac{\partial \psi_{\mu_{t}}}{\partial t}(z)\right|_{t=0} \frac{1}{\left(1+\psi_{\mu_{0}}(z)\right)^{2}} \\
& =-\frac{(1-z)^{2}}{z} \lim _{\varepsilon \rightarrow 0} \frac{\psi_{\mu_{\varepsilon}}(z)-\psi_{\mu_{0}}(z)}{\varepsilon} \\
& =-\frac{(1-z)^{2}}{z} \lim _{\varepsilon \rightarrow 0} \frac{1}{\varepsilon} \int_{\mathbf{C}}\left(\frac{z \tau}{1-z \tau}-\frac{z}{1-z}\right) d \mu_{\varepsilon}(\tau) .
\end{aligned}
$$

An easy algebraic manipulation concludes now the proof of the proposition.

6. Infinite divisibility on the circle. In this section we give a complete characterization of $\otimes$-infinitely divisible measures in $\mathscr{M}_{\mathbf{T}}$. First we will see that all of these measures, with the exception of normalized arclength measure $P_{0}$, belong to $\mathscr{M}_{*}$.

6.1. LemmA. If $\mu \in \mathscr{M}_{\mathbf{T}} \backslash \mathscr{M}_{*}$ is $\otimes$-infinitely divisible then $\mu=P_{0}$.

Proof. We can write $\mu=\nu \otimes \nu$ with $\nu \in \mathscr{M}_{\mathrm{T}}$ and, since $\left(\mathscr{M}_{*}, \otimes\right)$ is a group, we must have $\nu \in \mathscr{M}_{\mathbf{T}} \backslash \mathscr{M}_{*}$. By property (3) in $\S 1$ we have $\int_{\mathrm{T}} \zeta^{n} d \mu(\zeta)=0$ for $n=1,2, \ldots$, and the result follows from Lemma 2.1.

\subsection{Corollary. For every $\mu \in \mathscr{M}_{\mathrm{T}}$ we have $P_{0} \otimes \mu=P_{0}$.}

Proof. We have $P_{0} \otimes \mu=P_{0} \otimes\left(P_{0} \otimes \mu\right)=\nu_{1} \otimes \nu_{2}$ with $\nu_{1}, \nu_{2} \in$ $\mathscr{M}_{\mathrm{T}} \backslash \mathscr{M}_{*}$. The conclusion follows exactly as in the proof of Lemma 6.1 .

We concentrate now on measures $\mu \in \mathscr{M}_{\mathrm{T}} \cap \mathscr{M}_{*}$, and we start by generating a few interesting probability measures on the circle. The following result generates a measure analogous to the Gaussian distribution on the line.

6.3. LEMMA. For every $\gamma>0$, the function

$$
S(z)=\exp \left(\gamma\left(z+\frac{1}{2}\right)\right)
$$

is of the form $S=S_{\mu}$ for some $\mu \in \mathscr{M}_{\mathrm{T}} \cap \mathscr{M}_{*}$.

Proof. Given $\varepsilon \in(0,1 / 10)$, consider the measure $\mu_{\varepsilon} \in \mathscr{M}_{\mathbf{T}} \cap \mathscr{M}_{*}$ defined by $\mu_{\varepsilon}=\left(\delta_{\zeta}+\delta_{\bar{\zeta}}\right) / 2$, where $\zeta=\sqrt{1-\varepsilon}+i \sqrt{\varepsilon}$. By Lemma 
4.1, we have

$$
\begin{aligned}
S_{\mu_{\varepsilon}}(z) & =\frac{2(z+1)}{\sqrt{1-\varepsilon}(1+2 z)+\sqrt{(1-\varepsilon)(1+2 z)^{2}-4 z(z+1)}} \\
& =\frac{2(z+1)}{\sqrt{1-\varepsilon}(1+2 z)+\sqrt{1-\varepsilon(1+2 z)^{2}}} \\
& =\frac{2(z+1)}{(1-\varepsilon / 2)(1+2 z)+1-(\varepsilon / 2)(1+2 z)^{2}+O\left(\varepsilon^{2}\right)} \\
& =\frac{2(z+1)}{2(z+1)[1-\varepsilon(z+1 / 2)]+O\left(\varepsilon^{2}\right)} \\
& =1+\varepsilon\left(z+\frac{1}{2}\right)+O\left(\varepsilon^{2}\right),
\end{aligned}
$$

where the $O\left(\varepsilon^{2}\right)$ estimate is uniform for $|z| \leq 1 / 2$. Set $\varepsilon_{n}=\gamma / n$ and note that

$$
\lim _{n \rightarrow \infty} S_{\mu_{\varepsilon_{n}}}(z)^{n}=\exp \left(\gamma\left(z+\frac{1}{2}\right)\right)
$$

uniformly for $|z| \leq 1 / 2$. Since

$$
S_{\mu}(z)^{n}=S_{n \text { times }}^{S_{\mu \otimes \mu \otimes \cdots \otimes \mu}}(z),
$$

our lemma follows from Corollary 2.4.

Next we consider an analogue of the Poisson distribution on the line.

6.4. LeMmA. Fix $\gamma>0$ and $t \in \mathbf{R}$, and set

$$
S(z)=\exp \left(\frac{\gamma}{z+\frac{1}{2}+i t}\right) .
$$

Then there exists $\mu \in \mathscr{M}_{\mathbf{T}} \cap \mathscr{M}_{*}$ such that $S=S_{\mu}$.

Proof. Choose $\zeta \in \mathbf{T}$ such that $1 /(1-\zeta)=1 / 2+i t$, and for $\varepsilon \in(0,1 / 10)$ define $\mu_{\varepsilon}=(1-\varepsilon) \delta_{1}+\varepsilon \delta_{\zeta}$. We have

$$
S_{\mu_{\varepsilon}}(z)=\frac{2(z+1)}{1+z+\zeta z+\varepsilon(\zeta-1)+\sqrt{(1+z+\zeta z+\varepsilon(\zeta-1))^{2}-4 \zeta z(z+1)}}
$$

by Lemma 4.1 . Note that the polynomial

$$
p(z)=(1+z+\zeta z+\varepsilon(\zeta-1))^{2}-4 \zeta z(z+1)
$$


does not vanish for $|z| \leq 1 / 100$, and

$$
\begin{aligned}
\sqrt{p(z)} & =\sqrt{(1+z+\zeta z)^{2}-4 \zeta z(z+1)+2 \varepsilon(\zeta-1)(1+z+\zeta z)+O\left(\varepsilon^{2}\right)} \\
& =\sqrt{(1+z-\zeta z)^{2}+2 \varepsilon(\zeta-1)(1+z+\zeta z)+O\left(\varepsilon^{2}\right)} \\
& =1+z-\zeta z+\varepsilon(\zeta-1) \frac{1+z+\zeta z}{1+z-\zeta z}+O\left(\varepsilon^{2}\right),
\end{aligned}
$$

uniformly for $|z| \leq 1 / 100$. We deduce that

$$
\begin{aligned}
S_{\mu_{\varepsilon}}(z) & =\frac{2(z+1)}{2(z+1)+\varepsilon(\zeta-1)+\varepsilon(\zeta-1) \frac{1+z+\zeta z}{1+z-\zeta z}+O\left(\varepsilon^{2}\right)} \\
& =\frac{2(z+1)}{2(z+1)+\varepsilon(\zeta-1) \frac{2(z+1)}{1+z-\zeta z}+O\left(\varepsilon^{2}\right)} \\
& =1+\varepsilon \frac{1-\zeta}{1+z-\zeta z}+O\left(\varepsilon^{2}\right) \\
& =1+\varepsilon \frac{1}{z+\frac{1}{1-\zeta}}+O\left(\varepsilon^{2}\right) \\
& =1+\varepsilon \frac{1}{z+\frac{1}{2}+i t}+O\left(\varepsilon^{2}\right),
\end{aligned}
$$

uniformly for $|z| \leq 1 / 100$. The conclusion follows now as in the proof of Lemma 6.3.

Lemmas 6.3 and 6.4 have the following analogue for the functions $\Sigma_{\mu}$.

LemMA 6.5. For every $\gamma>0$, and every $\zeta \in \mathbf{T}$, the function

$$
\Sigma(z)=\exp \left(\gamma \frac{1+\zeta z}{1-\zeta z}\right)
$$

is of the form $\Sigma=\Sigma_{\mu}$ for some $\mu \in \mathscr{M}_{\mathrm{T}} \cap \mathscr{M}_{*}$.

Proof. It suffices to show that the function $S(z)=\Sigma(z /(z+1))$ has the form $S=S_{\mu}$ for some $\mu \in \mathscr{M}_{\mathrm{T}} \cap \mathscr{M}_{*}$. If $\zeta=1$ then $S(z)=$ $\exp (2 \gamma(z+1 / 2))$, and the result follows from Lemma 6.3. If $\zeta \neq 1$ then an easy calculation, using the fact that $1 / \zeta=\bar{\zeta}$, shows that

$$
\begin{aligned}
\frac{\zeta+z /(1+z)}{\zeta-z /(1+z)} & =\frac{-2 \zeta}{(\zeta-1)((\zeta-1) z+\zeta)}+\frac{\zeta+1}{\zeta-1} \\
& =\frac{2}{(\bar{\zeta}-1)((\zeta-1) z+\zeta)}+\frac{\zeta+1}{\zeta-1} \\
& =\frac{2}{|\zeta-1|^{2}} \frac{1}{z+\frac{\zeta}{\zeta-1}}+\frac{\zeta+1}{\zeta-1} .
\end{aligned}
$$


Note now that $\zeta /(\zeta-1)=1 / 2+i t$ and $(\zeta+1) /(\zeta-1)=i \alpha$ for some real numbers $t, \alpha$, so that

$$
S(z)=\exp (i \alpha) \exp \left(\gamma^{\prime} \frac{1}{z+\frac{1}{2}+i t}\right),
$$

with $\gamma^{\prime}=2 \gamma /|\zeta-1|^{2}$. The lemma follows now from Lemma 6.4.

We are now ready to study the $\otimes$-infinitely divisible measures in $\mathscr{M}_{\mathrm{T}} \cap \mathscr{M}_{*}$. First we relate them with free convolution semigroups. The following lemma has an obvious analogue for the function $\Sigma_{\mu}$.

6.6. Lemma. For every $\mu \in \mathscr{M}_{\mathrm{T}} \cap \mathscr{M}_{*}$ there exists a function $v(z)$, analytic with nonnegative real part in a neighborhood of $z=0$, such that $S_{\mu}(z)=\exp (v(z))$. If $\mu$ is $\otimes$-infinitely divisible, then for every $t \geq 0$ there exists $\mu_{t} \in \mathscr{M}_{\mathbf{T}} \cap \mathscr{M}_{*}$ such that $S_{\mu_{t}}(z)=\exp (t v(z))$ for sufficiently small $|z|$.

Proof. If $\left|S_{\mu}(0)\right|=1$, we have $\mu=\delta_{\exp (i \theta)}$ for some $\theta \in \mathbf{R}$. In this case $S_{\mu}(z)=\exp (-i \theta), \mu_{t}=\delta_{\exp (i t \theta)}$, and the lemma is obvious. If $\left|S_{\mu}(0)\right|>1$ then $\left|S_{\mu}(z)\right|>1$ in a neighborhood of $z=0$, whence the existence of the function $v(z)$ in such a neighborhood. Of course, the function $v(z)$ is not unique since it can be replaced by $v(z)+2 k \pi i$, $k \in \mathbf{Z}$.

Assume now that

$$
\mu=\underbrace{\nu \otimes \nu \otimes \cdots \otimes \nu}_{n \text { times }},
$$

and choose $w(z)$ such that $S_{\nu}(z)=\exp (w(z))$ for $|z|$ small. We have then $S_{\nu}(z)^{n}=S_{\mu}(z)$, and therefore $n w(z)=v(z)+2 k \pi i$ for $z$ in some neighborhood of zero, and $k \in \mathbf{Z}$. It is easy to see now that the measure $\nu^{\prime}=\nu \otimes \delta_{\alpha}$, where $\alpha=\exp (2 k \pi i / n)$, satisfies

$$
S_{\nu^{\prime}}(z)=\exp \left(w(z)-\frac{2 k \pi i}{n}\right)=\exp \left(\frac{1}{n} v(z)\right) .
$$

Assume now that $\mu$ is $\otimes$-infinitely divisible. The previous argument shows that $\exp (v(z) / n)$ has the form $S_{\nu_{n}}$ for some $\nu_{n} \in \mathscr{M}_{\mathrm{T}} \cap \mathscr{M}_{*}$, and hence $\exp (t v(z))=S_{\mu_{t}}(z)$ for some $\mu_{t} \in \mathscr{M}_{\mathrm{T}} \cap \mathscr{M}_{*}$ provided that $t$ is positive and rational. The conclusion of the lemma follows now from Corollary 2.4 .

The preceding lemma shows that for every $\nabla$-infinitely divisible measure $\mu \in \mathscr{M}_{\mathrm{T}} \cap \mathscr{M}_{*}$ there exists a one-parameter $\otimes$-semigroup $\left\{\mu_{t}: t \geq 0\right\}$ (i.e., $\mu_{t} \otimes \mu_{s}=\mu_{t+s}$ ) such that $\mu_{1}=\mu$. 
We are now able to give a complete characterization of the $\Delta$ infinitely divisible measures in $\mathscr{M}_{\mathrm{T}} \cap \mathscr{M}_{*}$, as well as an analogue of the Lévy-Hinčin formula from the classical theory of infinitely divisible distributions on the line. We formulate the result both in terms of $S_{\mu}$ and $\Sigma_{\mu}$.

6.7. Theorem. (1) A measure $\mu \in \mathscr{M}_{\mathbf{T}} \cap \mathscr{M}_{*}$ is $\otimes$-infinitely divisible if and only if there exists a function $v(z)$, analytic in $\{z: \Re z>$ $-1 / 2\}$, such that

$$
\Re v(z) \geq 0 \quad \text { if } \Re z>-\frac{1}{2},
$$

and $S_{\mu}(z)=\exp (v(z))$.

(2) A measure $\mu \in \mathscr{M}_{\mathbf{T}} \cap \mathscr{M}_{*}$ is $\otimes$-infinitely divisible if and only if there exists a function $u(z)$, analytic in $\mathbf{D}$, such that

$$
\Re u(z) \geq 0 \quad \text { if } z \in \mathbf{D},
$$

and $\Sigma_{\mu}(z)=\exp (u(z))$.

(3) If $v(z)$ is an analytic function in $\{z: \Re z>-1 / 2\}$, such that

$$
\Re v(z) \geq 0 \quad \text { if } \Re z>-\frac{1}{2},
$$

then the function $S(z)=\exp (v(z))$ has the form $S=S_{\mu}$ for some -infinitely divisible measure $\mu \in \mathscr{M}_{\mathbf{T}} \cap \mathscr{M}_{*}$.

(4) If $u(z)$ is an analytic function in $\mathbf{D}$, such that

$$
\Re u(z) \geq 0 \quad \text { if } z \in \mathbf{D},
$$

then the function $\Sigma(z)=\exp (u(z))$ has the form $\Sigma=\Sigma_{\mu}$ for some $\otimes$-infinitely divisible measure $\mu \in \mathscr{M}_{\mathrm{T}} \cap \mathscr{M}_{*}$.

(5) Let $\mu \in \mathscr{M}_{\mathrm{T}} \cap \mathscr{M}_{*}$ be a $\otimes$-infinitely divisible probability measure, let $u(z)$ be an analytic function with nonnegative real part in $\mathbf{D}$ such that $\Sigma_{\mu}(z)=\exp (u(z))$, and let $\left\{\mu_{t}: t \geq 0\right\} \subset \mathscr{M}_{\mathbf{T}} \cap \mathscr{M}_{*}$ satisfy $\Sigma_{\mu_{t}}(z)=\exp (t u(z))$ and $\mu_{1}=\mu$. Then the positive measures $\nu_{\varepsilon}$ defined by $d \nu_{\varepsilon}(\zeta)=(1 / \varepsilon)(1-\Re \zeta) d \mu_{\varepsilon}(\zeta)$ converge in the weak ${ }^{*}$ topology to a measure $\nu$ as $\varepsilon \rightarrow 0$, the limit $\alpha=\lim _{\varepsilon \rightarrow 0}(1 / \varepsilon) \int_{\mathbf{T}} \Im \zeta d \mu_{\varepsilon}(\zeta)$ exists, and

$$
u(z)=-i \alpha+\int_{\mathbf{T}} \frac{1+\zeta z}{1-\zeta z} d \nu(\zeta) \quad \text { if } z \in \mathbf{D}
$$

Proof. The equivalence between (1) and (2), and between (3) and (4), is seen by the change of variable $u(z)=v(z /(1-z))$. Therefore it 
will suffice to prove (2), (4), and (5). We start with (4), and we remark that it suffices to show that $\Sigma(z)=\exp (u(z))$ has the form $\Sigma=\Sigma_{\mu}$ for some $\mu \in \mathscr{M}_{\mathbf{T}} \cap \mathscr{M}_{*}$; such a $\mu$ must then be infinitely divisible since $\Sigma(z)=\Sigma_{n}(z)^{n}$, where $\Sigma_{n}(z)=\exp (u(z) / n)$. A theorem of Herglotz concerning analytic functions with nonnegative real part in the unit disc shows that a function $u$ with nonnegative real part in $\mathbf{D}$ can be written as

$$
u(z)=u_{\alpha, \nu}(z)=-i \alpha+\int_{\mathbf{T}} \frac{\zeta+z}{\zeta-z} d \nu(\zeta), \quad z \in \mathbf{D},
$$

for some finite measure $\nu$ on $\mathbf{T}$, and some real number $\alpha$. Since finite measures on $\mathbf{T}$ can be approximated in the weak* topology by measures with finite support, and since the family of functions with nonnegative real part in $\mathbf{D}$ is normal, we deduce that $u$ can be approximated uniformly on compact subsets of $\mathbf{D}$ by functions of the form $u_{\alpha, \nu^{\prime}}$, where $\nu^{\prime}$ has finite support. By Corollary 2.4, it suffices to prove (4) in the particular case when $\nu$ has finite support. In this particular case the conclusion follows immediately from Lemma 6.5.

Assume next that $\mu \in \mathscr{M}_{\mathbf{T}} \cap \mathscr{M}_{*}$ is $\otimes$-infinitely divisible, and let $\left\{\mu_{t}: t \geq 0\right\} \subset \mathscr{M}_{\mathbf{T}} \cap \mathscr{M}_{*}$ satisfy $\Sigma_{\mu_{t}}(z)=\exp (t u(z))$ and $\mu_{1}=\mu$, where $u(z)$ is a convergent power series. We would like to apply now Lemma 5.1, but we observe first that

$$
\frac{(1-\zeta)(1-z)}{1-\zeta z}=(1-\Re \zeta) \frac{1+\zeta z}{1-\zeta z}-i \Im \zeta
$$

for $\zeta \in \mathbf{T}$. Then Lemma 5.1 implies that

$$
\Re u(z)=\lim _{\varepsilon \rightarrow 0} \int_{\mathbf{T}} \frac{1-|z|^{2}}{|1-\zeta z|^{2}} d \nu_{\varepsilon}(\zeta)
$$

for $z$ sufficiently close to zero. Taking $z=0$, we see that the measures $\nu_{\varepsilon}$ remain bounded as $\varepsilon \rightarrow 0$. If $\nu$ is any cluster point of $\nu_{\varepsilon}$ as $\varepsilon \rightarrow 0$, we must have

$$
\Re u(z)=\int_{\mathbf{T}} \frac{1-|z|^{2}}{|1-\zeta z|^{2}} d \nu(\zeta) .
$$

This formula shows that in fact $\nu$ is unique, and hence it is the limit of the $\nu_{\varepsilon}$ as $\varepsilon \rightarrow 0$. Now, the formula in Lemma 5.1 can be written as

$$
u(z)=\lim _{\varepsilon \rightarrow 0}\left[\int_{\mathbf{T}} \frac{1+\zeta z}{1-\zeta z} d \nu_{\varepsilon}(\zeta)-\frac{i}{\varepsilon} \int_{\mathbf{T}} \Im \zeta d \mu_{\varepsilon}(\zeta)\right],
$$

and the above considerations show that

$$
\lim _{\varepsilon \rightarrow 0} \int_{\mathbf{T}} \frac{1+\zeta z}{1-\zeta z} d \nu_{\varepsilon}(\zeta)=\int_{\mathbf{T}} \frac{1+\zeta z}{1-\zeta z} d \nu(\zeta)
$$


It follows now immediately that the limit $\alpha=\lim _{\varepsilon \rightarrow 0}(1 / \varepsilon) \int_{\mathbf{T}} \Im \zeta d \mu_{\varepsilon}(\zeta)$ exists, and

$$
u(z)=-i \alpha+\int_{\mathrm{T}} \frac{1+\zeta z}{1-\zeta z} d \nu(\zeta)
$$

for $z$ close to zero. It is clear now that $u$ has an analytic continuation, with nonnegative real part, to the entire disc $\mathbf{D}$. This concludes the proof of (2) and (5).

We conclude this section with a description of some properties of $\otimes$-infinitely divisible measures in $\mathscr{M}_{\mathrm{T}} \cap \mathscr{M}_{*}$.

6.8. Proposition. Let $\mu \in \mathscr{M}_{\mathrm{T}} \cap \mathscr{M}_{*}$ be a $\otimes$-infinitely divisible probability measure, and let $\left\{\mu_{t}: t \geq 0\right\} \subset \mathscr{M}_{\mathrm{T}} \cap \mathscr{M}_{*}$ be such that $\mu_{1}=\mu$ and $\Sigma_{\mu_{t}}(z)=\exp (t u(z))$, where $u$ is analytic with nonnegative real part in $\mathbf{D}$. Denote

$$
\psi(t, z)=\psi_{\mu_{t}}^{\sim}(z), \quad \chi(t, z)=\chi_{\mu_{t}}^{\sim}(z), \quad t \geq 0 ;
$$

note that $\psi(t, z)$ and $\chi(t, z)$ are defined for all $z \in \mathbf{D}$.

(1) The map $t \mapsto \mu_{t}$ is continuous for $t \geq 0$ when $\mathscr{M}_{\mathrm{T}}$ is endowed with the weak ${ }^{*}$ topology.

(2) For all $t \geq 0$ and $z \in \mathbf{D}$ we have $\chi(t, \psi(t, z))=z$.

(3) For all $t \geq 0$ we have

$$
\{\psi(t, z):|z|<1\}=\{z: z \in \mathbf{D},|\chi(t, z)|<1\} .
$$

Proof. (1) This assertion follows immediately from Proposition 2.9.

(2) We know that $|\psi(t, z)|<1$ for all $t \geq 0$ and $z \in \mathbf{D}$. Therefore the expression $\chi(t, \psi(t, z))$ makes sense for all such $t$ and $z$. For fixed $t$, we have $\chi(t, \psi(t, z))=z$ for small $z$, and (2) follows because of the uniqueness of analytic continuation.

(3) Fix $t \geq 0$, and note that the inclusion

$$
\{\psi(t, z):|z|<1\} \subset\{z: z \in \mathbf{D},|\chi(t, z)|<1\}
$$

follows immediately from (2). To prove the opposite inclusion take any $z \in \mathbf{D}$ such that $|\chi(t, z)|<1$. It suffices to show that for such a point $z$ we have $\psi(t, \chi(t, z))=z$. Since

$$
\chi(t, z)=z \exp (t u(z)) /(z+1),
$$

it follows that $|\chi(s, z)|<1$ for all $s \in[0, t]$. The set $A=\{s \in[0, t]$ : $\psi(s, \chi(s, z))=z\}$ is closed and $0 \in A$. We conclude the proof by showing that $A$ is open in $[0, t]$, and hence $t \in A$ by connectedness. 
Indeed, if $s_{0} \in A$ then $z$ belongs to the range of $\psi\left(s_{0}, w\right)$. Since $\psi\left(s_{0}, w\right)=\lim _{s \rightarrow s_{0}} \psi(s, w)$ uniformly for $w$ in a compact subset of the unit disc, $z$ must belong to the range of $\psi(s, w)$ for $s$ close to $s_{0}$, and hence by (2) we have $\psi(s, \chi(s, z))=z$ for $s$ close to $s_{0}$.

7. Infinite divisibility on the positive axis. In this section we characterize the $\otimes$-infinitely divisible probability measures in $\mathscr{M}_{\mathbf{R}_{+}} \cdot \operatorname{Re}$ call that the measures in $\mathscr{M}_{\mathbf{R}_{+}}$have compact supports, contained in $(0,+\infty)$. We begin with analogues of Lemmas $6.3,6.4$, and 6.5.

7.1. LeMma. For every $\gamma>0$ the function

$$
S(z)=\exp \left(-\gamma\left(z+\frac{1}{2}\right)\right)
$$

is of the form $S=S_{\mu}$ for some $\mu \in \mathscr{M}_{\mathbf{R}_{+}}$.

Proof. Given $\varepsilon \in(0,1 / 10)$, consider the measure $\mu_{\varepsilon}=\left(\delta_{a}+\delta_{b}\right) / 2$, where $a=1+\sqrt{2 \varepsilon}$ and $b=1 / a$. Since $a b=1$ and $a+b=$ $2+2 \varepsilon+o(\varepsilon)$, we see that

$$
\begin{aligned}
S_{\mu_{\varepsilon}}(z) & =\frac{2(z+1)}{(1+\varepsilon)(2 z+1)+\sqrt{(1+\varepsilon)^{2}(2 z+1)^{2}-4 z(z+1)}+o(\varepsilon)} \\
& =\frac{2(z+1)}{(1+\varepsilon)(2 z+1)+\sqrt{1+2 \varepsilon(2 z+1)^{2}}+o(\varepsilon)} \\
& =\frac{2(z+1)}{(1+\varepsilon)(2 z+1)+1+\varepsilon(2 z+1)^{2}+o(\varepsilon)} \\
& =\frac{2(z+1)}{2(z+1)(1+\varepsilon(2 z+1))+o(\varepsilon)} . \\
& =1-\varepsilon(2 z+1)+o(\varepsilon)
\end{aligned}
$$

uniformly for sufficiently small $z$. The proof is completed as in Lemma 6.1, using Corollary 3.2 in place of Corollary 2.4.

7.2. LemMA. Fix $\gamma>0$ and $t \in \mathbf{R} \backslash[0,1]$, and set

$$
S(z)=\exp \left(\frac{\gamma}{z+t}\right) \text {. }
$$

Then there exists $\mu \in \mathscr{M}_{\mathbf{R}_{+}}$such that $S=S_{\mu}$.

Proof. Choose $\zeta \in \mathbf{R}_{+}$such that $t=1 /(1-\zeta)$, and for $\varepsilon$ sufficiently small define $\mu_{\varepsilon}=(1-\varepsilon) \delta_{1}+\varepsilon \delta_{\zeta}$. The rest of the proof is virtually identical with that of Lemma 11, except that one must use Corollary 3.2 in place of Corollary 2.4 . 
7.3. Lemma. For every $\gamma>0$ and every $t \in \mathbf{R}_{+}$, the function

$$
\Sigma(z)=\exp \left(-\gamma \frac{z}{1-t z}\right)
$$

is of the form $\Sigma=\Sigma_{\mu}$ for some $\mu \in \mathscr{M}_{\mathbf{R}_{+}}$.

Proof. It suffices to show that the function $S(z)=\Sigma(z /(1+z))$ is of the form $S=S_{\mu}$ for some $\mu \in \mathscr{M}_{\mathbf{R}_{+}}$. When $t=1$ we have $S(z)=\exp (-\gamma z)$, and the result follows from Lemma 7.1. When $t \neq 1$ an easy calculation shows that $S(z)=\alpha \exp \left(\gamma^{\prime} /\left(z+t^{\prime}\right)\right)$, where $\alpha=\exp (-\gamma /(1-t)), t^{\prime}=1 /(1-t)$, and $\gamma^{\prime}=\gamma /(1-t)^{2}$. In this case the results follow from Lemma 7.2.

The analogue of Lemma 6.6 is easier to prove for measures in $\mathscr{M}_{\mathbf{R}_{+}}$. Again, we do not give the obvious statement for the function $\Sigma_{\mu}$.

7.4. Lemma. For every $\mu \in \mathscr{M}_{\mathbf{R}_{+}}$there exists a function $v(z)$, analytic for $z$ in a neighborhood of $[-1,0]$ such that $v(\bar{z})=\overline{v(z)}$, $v^{\prime}(z) \leq 0$ for $z \in[-1,0]$, and $S_{\mu}(z)=\exp (v(z))$. If $\mu$ is $\otimes-$ infinitely divisible, then for every $t \geq 0$ there exists $\mu_{t} \in \mathscr{M}_{\mathbf{R}_{+}}$such that $S_{\mu_{t}}(z)=\exp (t v(z))$ for $z$ in some neighborhood of $[-1,0]$. Moreover, for every $T>0$, the measures $\left\{\mu_{t}: 0 \leq t \leq T\right\}$ have supports bounded away from zero and infinity.

Proof. The existence of the function $v(z)$ is immediate from Proposition 3.1. Assume that

$$
\mu=\underbrace{\nu \otimes \nu \otimes \cdots \otimes \nu}_{n \text { times }}
$$

for some natural number $n$, and choose $w(z)$ such that $w(\bar{z})=\overline{w(z)}$ and $S_{\nu}(z)=\exp (w(z))$. We have then $S_{\nu}(z)^{n}=S_{\mu}(z)$, and therefore $w(z)=v(z) / n$ in some neighborhood of $[-1,0]$. The existence of $\mu_{t}$ for all $t \geq 0$ follows as in Lemma 6.6, with Corollary 3.2 in place of Corollary 2.4. The last assertion of the lemma is a consequence of Proposition 3.4.

We are now ready for the main result of this section.

7.5. THEOREM. (1) A measure $\mu \in \mathscr{M}_{\mathbf{R}_{+}}$is $\bigotimes$-infinitely divisible if and only if there exists a function $v(z)$, analytic in a neighborhood of $(\mathbf{C} \backslash \mathbf{R}) \cup[-1,0]$, such that $v(\bar{z})=\overline{v(z)}, \Im v(z) \leq 0$ for $\Im z>0$, and $S_{\mu}(z)=\exp (v(z))$. 
(2) A measure $\mu \in \mathscr{M}_{\mathbf{R}_{+}}$is $\otimes$-infinitely divisible if and only if there exists a function $u(z)$, analytic in a neighborhood of $\left(\mathbf{R} \backslash \mathbf{R}_{+}\right) \cup$ $\{\infty\}$, such that $u(\bar{z})=\overline{u(z)}, \Im u(z) \leq 0$ for $\Im z>0$, and $\Sigma_{\mu}(z)=$ $\exp (u(z))$.

(3) If $v(z)$ is an analytic function in a neighborhood of $(\mathbf{C} \backslash \mathbf{R}) \cup$ $[-1,0]$, such that $v(\bar{z})=\overline{v(z)}$, and $\Im v(z) \leq 0$ for $\Im z>0$, then the function $S(z)=\exp (v(z))$ has the form $S=S_{\mu}$ for some $\otimes$-infinitely divisible measure $\mu \in \mathscr{M}_{\mathbf{R}_{+}}$.

(4) If $u(z)$ is an analytic function in a neighborhood of $\left(\mathbf{R} \backslash \mathbf{R}_{+}\right) \cup$ $\{\infty\}$, such that $u(\bar{z})=\overline{u(z)}$, and $\Im u(z) \leq 0$ for $\Im z>0$, then the function $\Sigma(z)=\exp (u(z))$ has the form $\Sigma=\Sigma_{\mu}$ for some $\otimes$-infinitely divisible measure $\mu \in \mathscr{M}_{\mathbf{R}_{+}}$.

(5) Let $\mu \in \mathscr{M}_{\mathbf{R}_{+}}$be a $\otimes$-infinitely divisible probability measure, let $u(z)$ be an analytic function in a neighborhood of $\left(\mathbf{R} \backslash \mathbf{R}_{+}\right) \cup\{\infty\}$, satisfying $u(\bar{z})=\overline{u(z)}$, and $\Im u(z) \leq 0$ for $\Im z>0$, such that $\Sigma_{\mu}(z)=$ $\exp (u(z))$, and let $\left\{\mu_{t}: t \geq 0\right\} \subset \mathscr{M}_{\mathbf{R}_{+}}$satisfy $\Sigma_{\mu_{t}}(z)=\exp (t u(z))$ and $\mu_{1}=\mu$. Then the positive measures $\nu_{\varepsilon}$ defined by $d \nu_{\varepsilon}(t)=$ $(1 / \varepsilon)(1-t)^{2} d \mu_{\varepsilon}(t)$ converge in the weak* topology to a measure $\nu$ as $\varepsilon \rightarrow 0$, the limit $\alpha=\lim _{\varepsilon \rightarrow 0}(1 / \varepsilon) \int_{\mathbf{R}}(1-t) d \mu_{\varepsilon}(t)$ exists, and

$$
u(z)=\alpha-\int_{\mathbf{R}} \frac{z}{1-t z} d \nu(t), \quad z \in \mathbf{R} \backslash \mathbf{R}_{+} .
$$

Proof. As in the proof of Theorem 6.7, it suffices to prove (2), (4), and (5). We prove first (4); assume that the function $u$ satisfies the assumptions of (4). Then the Herglotz theorem can be transferred to the upper half plane to show the existence of a positive measure $\nu$, with compact support in $\mathbf{R}_{+}$, and of a real number $\alpha$, such that

$$
u(z)=u_{\alpha, \nu}(z)=\alpha-\int_{\mathbf{R}} \frac{z}{1-t z} d \nu(t), \quad z \in \mathbf{R} \backslash \mathbf{R}_{+} .
$$

Proposition 3.4 allows us to consider only the case in which the measure $\nu$ has finite support, and in that particular case the conclusion follows from Lemma 7.3.

Assume next that $\mu \in \mathscr{M}_{\mathbf{R}_{+}}$is $\nabla$-infinitely divisible, and let $\left\{\mu_{t}: t \geq\right.$ $0\} \subset \mathscr{M}_{\mathbf{R}_{+}}$satisfy $\Sigma_{\mu_{t}}(z)=\exp (t u(z))$ and $\mu_{1}=\mu$, where $u(z)$ is analytic in some neighborhood of $[-\infty, 0]$. Observe that

$$
\frac{(1-t)(1-z)}{1-t z}=1-t+\frac{(1-t)^{2} z}{1-t z}
$$


which shows by Lemma 5.1 that

$$
\Im u\left(\frac{z}{1-z}\right)=\lim _{\varepsilon \rightarrow 0} \int_{\mathbf{R}_{+}} \frac{-\Im z}{|1-t z|^{2}} d \nu_{\varepsilon}(t) .
$$

Lemma 7.4 shows that the measures $\nu_{\varepsilon}$ have supports bounded away from zero and infinity as $\varepsilon \rightarrow 0$, and the preceding formula is seen to imply the existence of the weak*-limit of $\nu_{\varepsilon}$ as in the proof of Theorem 6.7. The interested reader will have no difficulty completing the proof of (2) and (5).

We conclude the section with an analogue of Proposition 6.8.

7.6. Proposition. Let $\mu \in \mathscr{M}_{\mathbf{R}_{+}}$be a $\otimes$-infinitely divisible probability measure, and let $\left\{\mu_{t}: t \geq 0\right\} \subset \mathscr{M}_{\mathbf{R}_{+}}$be such that $\Sigma_{\mu_{t}}(z)=$ $\exp (t u(z))$ and $\mu_{1}=\mu$. Denote

$$
\psi(t, z)=\psi_{\mu_{t}}^{\sim}(z), \quad \chi(t, z)=\chi_{\mu_{t}}^{\sim}(z), \quad t \geq 0 ;
$$

note that $\psi(t, z)$ and $\chi(t, z)$ are defined in a neighborhood of $\left(\mathbf{R} \backslash \mathbf{R}_{+}\right) \cup\{\infty\}$ which may depend on $t$.

(1) The map $t \mapsto \mu_{t}$ is continuous for $t \geq 0$ when $\mathscr{M}_{\mathbf{R}_{+}}$is endowed with the weak* topology.

(2) For all $t \geq 0$ and $z \in \mathbf{C} \backslash \mathbf{R}_{+}$we have $\chi(t, \psi(t, z))=z$.

(3) For all $t>0$ the set $\{\psi(t, z): z \in \mathbf{C}, \Im z>0\}$ is a connected component of $\{z: z \in \mathbf{C}, \Im z>0, \Im \chi(t, z)>0\}$.

Proof. (1) This assertion follows immediately from Proposition 3.4.

(2) We have $\Im \psi(z, t)>0$ if $\Im z>0$, and therefore the expression $\chi(t, \psi(z, t))$ makes sense for such values of $z$. The conclusion follows from the uniqueness of analytic continuation, as in the proof of Proposition 6.8(2).

(3) The set $\{\psi(t, z): z \in \mathbf{C}, \Im z>0\}$ is connected, and by (2) it is contained in $\{z: z \in \mathbf{C}, \Im z>0, \Im \chi(t, z)>0\}$. The equality $\psi(t, \chi(t, z))=z$ holds for every $z \in\{\psi(t, z): z \in \mathbf{C}, \Im z>0\}$, and by analytic continuation it holds for all $z$ in the connected component of $\{z: z \in \mathbf{C}, \Im z>0, \Im \chi(t, z)>0\}$ containing $\{\psi(t, z): z \in$ C, $\Im z>0\}$. The result follows at once from these remarks.

8. Infinite divisibility for additive free convolution. The structure of $\boxplus$-infinitely divisible distributions on the real line was described in [6], and it was showed in [9] why this characterization is an analogue of the classical Lévy-Hinčin formula. In this section we provide a 
simpler derivation of these results, and we show how to obtain the analogue of the Lévy-Hinčin measure by a limiting process. We will make the exposition as self-contained as possible.

We start by reviewing certain basic facts from [6]. Given a compactly supported probability measure $\mu$ on $\mathbf{R}$, one constructs first the Cauchy transform $G_{\mu}(z)=\int_{\mathbf{R}} 1 /(z-t) d \mu(t)$, and a meromorphic function $K_{\mu}(z)$ such that $G_{\mu}\left(K_{\mu}(z)\right)=z$ in a neighborhood of $z=0$. Finally, one considers the function $\mathscr{R}_{\mu}(z)=K_{\mu}(z)-z^{-1}$. It was shown in [6] that $\mathscr{R}_{\mu}=\mathscr{R}_{\mu_{1}}+\mathscr{R}_{\mu_{2}}$ if $\mu=\mu_{1} \boxplus \mu_{2}$. We recall that $\mathscr{M}_{\mathbf{R}}$ denotes the collection of compactly supported probability measures on $\mathbf{R}$.

8.1. Proposition. Given a compact interval $[-a, a] \subset \mathbf{R}$, there exist a neighborhood $U$ of $\infty$ and a neighborhood $V$ of zero with the following property: for every $\mu \in \mathscr{M}_{\mathbf{R}}$ with $\operatorname{supp}(\mu) \subset[-a, a]$, the function $G_{\mu}$ is one-to-one on $U$, and $G_{\mu}(U) \supset V$. Thus $\mathscr{R}_{\mu}$ is defined on $V$, and $K_{\mu}(V) \subset U$.

Proof. Let $\mu \in \mathscr{M}_{\mathbf{R}}$ be supported in $[-a, a]$, and consider the function $f(z)=G_{\mu}(1 / z)$. Since $f^{\prime}(z)=\int_{-a}^{a} 1 /(1-t z)^{2} d \mu(t)$, it is easily seen that there exists a positive number $\varepsilon$, independent of $\mu$, such that $\Re f^{\prime}(z)>0$ for $|z|<\varepsilon$. For such an $\varepsilon$, the function $f(z)$ is one-to-one for $|z|<\varepsilon$. Furthermore,

$$
f(z)-z=\int_{-a}^{a} t z^{2} /(1-t z) d \mu(t)
$$

and this implies the inequality $|f(z)-z|<|z| / 2$ for $|z|<\varepsilon$ provided that $\varepsilon$ is sufficiently small. For such a value of $\varepsilon$ we have $\{f(z)$ : $|z|<\varepsilon\} \supset\{z:|z|<\varepsilon / 2\}$. The proposition follows now with $U=$ $\{z:|z|>\varepsilon\} \cup\{\infty\}$ and $V=\{z:|z|<\varepsilon / 2\}$.

In the following proof we use the elementary fact that an analytic function $f$ in a convex set, such that $\Re f^{\prime}(z)>0$ for all $z$, is one-toone. This is immediately seen from the formula

$$
(f(b)-f(a)) /(b-a)=\int_{0}^{1} f^{\prime}(a+t(b-a)) d t .
$$

8.2. Proposition. Given a sequence $\left\{\mu_{n}: n \geq 1\right\} \subset \mathscr{M}_{\mathbf{R}}$, the following assertions are equivalent:

(1) The supports of the measures $\mu_{n}$ are contained in a compact interval, and the sequence $\left\{\mu_{n}: n \geq 1\right\}$ converges in the weak* topology to a measure $\mu \in \mathscr{M}_{\mathbf{R}}$. 
(2) The sequence $\left\{\mathscr{R}_{\mu_{n}}: n \geq 1\right\}$ converges uniformly in some neighborhood of zero to a function $\mathscr{R}$.

If (1) and (2) are satisfied, then $\mathscr{R}=\mathscr{R}_{\mu}$.

Proof. Assume first that (1) is satisfied. Proposition 8.1 implies the existence of open sets $U$ and $V$ such that $K_{\mu_{n}}(V) \subset U$ for all $n$. We deduce immediately that the family $\left\{K_{\mu_{n}}: n \geq 1\right\}$, and hence the family $\left\{\mathscr{R}_{\mu_{n}}: n \geq 1\right\}$ is normal in $V$. To prove (2) it suffices therefore to show that the Taylor coefficients of $\mathscr{R}_{\mu_{n}}$ converge to the corresponding coefficients of $\mathscr{R}_{\mu}$. This last assertion is verified as in the proof of Proposition 2.5.

Assume now that (2) is satisfied. It suffices to show that the measures $\left\{\mu_{n}: n \geq 1\right\}$ are supported in a compact interval; the convergence assertion follows from the weak*-compactness of the collection of measures supported in a fixed compact interval, and from the first part of the proof. To do this we consider the functions $g_{n}(z)=1 / K_{\mu_{n}}(z)$. We have $g_{n}^{\prime}(z)=\left(1-z^{2} \mathscr{R}_{\mu_{n}}^{\prime}(z)\right) /\left(1+z \mathscr{R}_{\mu_{n}}(z)\right)^{2}$, and we deduce the existence of $\varepsilon>0$ such that $\Re g_{n}^{\prime}(z)>0$ for $|z|<\varepsilon$ and for all $n$. Moreover, $g_{n}(z)-z=-z^{2} \mathscr{R}_{\mu_{n}}(z) /\left(1+z \mathscr{R}_{\mu_{n}}(z)\right)$, and we see that for $\varepsilon$ small enough $g_{n}$ has an inverse function defined for $|z|<\varepsilon / 2$. We conclude that the function $G_{\mu_{n}}(z)$ can be continued analytically to $|z|>2 / \varepsilon$, and therefore $\operatorname{supp}\left(\mu_{n}\right) \subset[-2 / \varepsilon, 2 / \varepsilon]$ for all $n$.

The last assertion of the proposition follows from the first part of this proof.

The following calculations can be found in [9]. We present them here for the sake of completeness. They provide the free additive convolution analogues of the Gaussian measure (obtained by the de Moivre procedure), and of the Poisson measures.

8.3. LemMA. For every $\gamma>0$, the function $\mathscr{R}(z)=\gamma z$ is of the form $\mathscr{R}=\mathscr{R}_{\mu}$ for some $\mu \in \mathscr{M}_{\mathbf{R}}$.

Proof. Set $\varepsilon_{n}=\gamma / \sqrt{n}$, and consider the measures

$$
\mu_{n}=\left(\delta_{-\varepsilon_{n}}+\delta_{\varepsilon_{n}}\right) / 2, \quad n \geq 1 .
$$

A straightforward calculation shows that

$$
\mathscr{R}_{\mu_{n}}(z)=\left(-1+\sqrt{1+4 w^{2} \varepsilon_{n}^{2}}\right) / 2 w=w \varepsilon_{n}^{2}+O\left(\varepsilon_{n}^{4}\right),
$$

and therefore $n \mathscr{R}_{\mu_{n}}$ converge uniformly in some neighborhood of zero to $\mathscr{R}$. The lemma follows from the preceding proposition. 
8.4. LemMA. For every $\gamma>0$, and every $t \in \mathbf{R}, t \neq 0$, the function $\mathscr{R}(z)=\gamma z /(1-t z)$ is of the form $\mathscr{R}=\mathscr{R}_{\mu}$ for some $\mu \in \mathscr{M}_{\mathbf{R}}$.

Proof. Set $\varepsilon_{n}=\gamma / n$, and consider the measures $\mu_{n}=\left(1-\varepsilon_{n}\right) \delta_{0}+$ $\varepsilon_{n} \delta_{t}, n \geq 1$. A calculation shows that

$$
\begin{aligned}
\mathscr{R}_{\mu_{n}}(z) & =\frac{-1+t z+\sqrt{(1-t z)^{2}+4 t \varepsilon_{n} z}}{2 z} \\
& =\frac{-1+t z+(1+t z)\left(1+\frac{2 t \varepsilon_{n} z}{(1-t z)^{2}}\right)}{2 z}+O\left(\varepsilon_{n}^{2}\right) \\
& =\frac{t \varepsilon_{n} z}{1-t z}+O\left(\varepsilon_{n}^{2}\right),
\end{aligned}
$$

so that $n \mathscr{R}_{\mu_{n}}$ converge uniformly in some neighborhood of zero to $\mathscr{R}$.

We can now turn to the study of $\boxplus$-infinitely divisible measures in $\mathscr{M}_{\mathbf{R}}$. The following result is analogous to Lemma 7.4, and the reader will have no difficulty to verify it using Proposition 8.2.

8.5. LemMA. Let $\mu \in \mathscr{M}_{\mathbf{R}}$ be an $\boxplus$-infinitely divisible measure. For every $t \geq 0$ there exists a measure $\mu_{t} \in \mathscr{M}_{\mathbf{R}}$ such that $\mathscr{R}_{\mu_{t}}=$ $t \mathscr{R}_{\mu}$. Moreover, for every $T>0$, the measures $\left\{\mu_{t}: 0 \leq t \leq T\right\}$ have uniformly bounded supports.

The following theorem is the main result of this section.

8.6. THEOREM. (1) A measure $\mu \in \mathscr{M}_{\mathbf{R}}$ is $\boxplus$-infinitely divisible if and only if the function $\mathscr{R}_{\mu}$ can be continued analytically to a neighborhood of $(\mathbf{C} \backslash \mathbf{R}) \cup\{0\}$, such that $\Im \mathscr{R}_{\mu}(z) \geq 0$ for $\Im z>0$.

(2) Let $\mathscr{R}$ be an analytic function in a neighborhood of $(\mathbf{C} \backslash \mathbf{R}) \cup\{0\}$ such that $\mathscr{R}(\bar{z})=\overline{\mathscr{R}(z)}$, and $\Im \mathscr{R}(z) \geq 0$ for $\Im z>0$. Then $\mathscr{R}=\mathscr{R}_{\mu}$ for some $\boxplus$-infinitely divisible measure $\mu \in \mathscr{M}_{\mathbf{R}}$.

(3) Let $\mu \in \mathscr{M}_{\mathbf{R}}$ be $\boxplus$-infinitely divisible, and let $\left\{\mu_{t}: t \geq 0\right\} \subset \mathscr{M}_{\mathbf{R}}$ be such that $\mathscr{R}_{\mu_{t}}(z)=t \mathscr{R}_{\mu}(z)$. Then the positive measures $\nu_{\varepsilon}$ defined by $d \nu_{\varepsilon}(t)=(1 / \varepsilon) t^{2} d \mu_{\varepsilon}(t)$ converge in the weak $k^{*}$ topology to a measure $\nu$ as $\varepsilon \rightarrow 0$, the limit

$$
\alpha=\lim _{\varepsilon \rightarrow 0}(1 / \varepsilon) \int_{\mathbf{R}} t d \mu_{\varepsilon}(t)
$$

exists, and

for all $z \in(\mathbf{C} \backslash \mathbf{R}) \cup\{0\}$.

$$
\mathscr{R}_{\mu}(z)=\alpha+\int_{\mathbf{R}} \frac{z}{1-t z} d \nu(t)
$$


Proof. The proof of (2) follows from Lemmas 8.3 and 8.4, and Proposition 8.2. Indeed, any function $\mathscr{R}$, satisfying the hypotheses of (2), can be written as

$$
\mathscr{R}(z)=\alpha+\int_{\mathbf{R}} \frac{z}{1-t z} d \nu(t)
$$

for $z \in(\mathbf{C} \backslash \mathbf{R}) \cup\{0\}$, where $\alpha$ is real and $\nu \in \mathscr{M}_{\mathbf{R}}$. Approximating $\nu$ in the weak* topology with measures with finite support, we see that $\mathscr{R}=\mathscr{R}_{\mu}$ for some $\mu$. The $\boxplus$-infinite divisibility of $\mu$ follows from the same argument applied to $\mathscr{R} / n, n=2,3, \ldots$.

Assume now that $\mu \in \mathscr{M}_{\mathbf{R}}$ is $\boxplus$-infinitely divisible, and $\left\{\mu_{t}: t \geq 0\right\}$ $\subset \mathscr{M}_{\mathbf{R}}$ are such that $\mathscr{R}_{\mu_{t}}(z)=t \mathscr{R}_{\mu}(z)$. Let us denote $G(t, z)=$ $G_{\mu_{t}}(z)$. It was shown in [6] that

$$
\frac{\partial G}{\partial t}\left(0, \frac{1}{z}\right)+\frac{\partial G}{\partial z}\left(0, \frac{1}{z}\right) \mathscr{R}_{\mu}(z)=0
$$

for $z$ close to zero. Since $G(0, z)=1 / z$, we have $(\partial G / \partial z)(0,1 / z)$ $=-z^{2}$, and therefore

$$
\begin{aligned}
\mathscr{R}_{\mu}(z) & =z^{-2} \lim _{\varepsilon \rightarrow 0} \frac{1}{\varepsilon}\left[G\left(\varepsilon, \frac{1}{z}\right)-G\left(0, \frac{1}{z}\right)\right] \\
& =z^{-2} \lim _{\varepsilon \rightarrow 0} \frac{1}{\varepsilon}\left[\int_{\mathbf{R}} \frac{z}{1-t z} d \mu_{\varepsilon}(t)-z\right] \\
& =\lim _{\varepsilon \rightarrow 0} \frac{1}{\varepsilon} \int_{\mathbf{R}} \frac{t}{1-t z} d \mu_{\varepsilon}(t) .
\end{aligned}
$$

Now, we have

$$
\frac{t}{1-t z}=t+\frac{t^{2} z}{1-t z}
$$

and we see that

$$
\Im \mathscr{R}_{\mu}(z)=\lim _{\varepsilon \rightarrow 0} \int_{\mathbf{R}} \frac{\Im z}{|1-t z|^{2}} d \nu_{\varepsilon}(t) .
$$

By Proposition 8.5, the measures $\mu_{\varepsilon}$, and hence $\nu_{\varepsilon}$ as well, have bounded supports as $\varepsilon \rightarrow 0$. The rest of the proof is virtually identical with that of Theorems 6.7 and 7.5. 


\section{REFERENCES}

[1] N. I. Achieser, The classical moment problem, in Russian, Fizmatgiz, Moscow, 1961.

[2] W. Feller, An Introduction to Probability Theory and its Applications, vol. II, John Wiley \& Sons, New York, 1971.

[3] B. V. Gnedenko and A. N. Kolmogorov, Limit Distributions for Sums of Independent Random Variables, Addison-Wesley Publishing Company, Cambridge, Massachusetts, 1954.

[4] P. Lévy, Théorie de l'Addition des Variables Aléatoires, Gauthier-Villars, Paris, 1937.

[5] D. Voiculescu, Symmetries of Some Reduced Free Product $C^{*}$-Algebras, in Operator Algebras and Their Connections with Topology and Ergodic Theory, Lecture Notes in Math., No. 1132, Springer Verlag, New York, 1985, pp. 556-588.

[6] , Addition of certain non-commuting random variables, J. Funct. Anal., 66 (1986), 323-346.

[7] Multiplication of certain non-commuting random variables, J. Operator Theory, 18 (1987), 223-235.

[8] _ Dual algebraic structures on operator algebras related to free products, J. Operator Theory, 18 (1987), 85-98.

[9] - Free noncommutative random variables, random matrices and the $\mathrm{II}_{1}$ factors of free groups, preprint, 1990.

Received December 1, 1990. Both authors were partially supported by grants from the National Science Foundation. The first named author was also supported by a grant from the Williford Energy Corporation.

INDIANA UNIVERSITY

BLOOMINGTON, IN 47405

AND

UNIVERSITY OF CALIFORNIA

BERKELEY, CA 94720 


\section{PACIFIC JOURNAL OF MATHEMATICS EDITORS}

\author{
V. S. VARADARAJAN \\ (Managing Editor) \\ University of California \\ Los Angeles, CA 90024-1555-05 \\ Herbert Clemens \\ University of Utah \\ Salt Lake City, UT 84112 \\ F. Michael Christ \\ University of California \\ Los Angeles, CA 90024-1555 \\ THOMAS ENRIGHT \\ University of California, San Diego \\ La Jolla, CA 92093
}

Nicholas ERCOLANI

University of Arizona

Tucson, AZ 85721

R. FINN

Stanford University

Stanford, CA 94305

VAUGHAN F. R. JONES

University of California

Berkeley, CA 94720

Steven Kerckhoff

Stanford University

Stanford, CA 94305

\section{C. MOORE}

University of California

Berkeley, CA 94720

Martin ScharlemanN

University of California

Santa Barbara, CA 93106

HAROLD STARK

University of California, San Diego

La Jolla, CA 92093

\section{R. ARENS \\ ASSOCIATE EDITORS}

\begin{tabular}{|c|c|c|c|c|}
\hline R. ARENS & $\begin{array}{l}\text { E. F. BECKENBACH } \\
(1906-1982)\end{array}$ & B. H. NeumanN & $\begin{array}{c}\text { F. WolF } \\
(1904-1989)\end{array}$ & K. YoshidA \\
\hline \multicolumn{5}{|c|}{ SUPPORTING INSTITUTIONS } \\
\hline \multicolumn{2}{|c|}{ UNIVERSITY OF ARIZONA } & UNIVER: & OF OREG & \\
\hline \multicolumn{2}{|c|}{ UNIVERSITY OF BRITISH COLUMB } & & & \\
\hline \multicolumn{2}{|c|}{ CALIFORNIA INSTITUTE OF TECHNOLOGY } & OGY STANI & INIV & \\
\hline \multicolumn{2}{|c|}{ UNIVERSITY OF CALIFORNIA } & UNI & & \\
\hline \multicolumn{2}{|c|}{ MONTANA STATE UNIVERSITY } & UNIVE & OF T & \\
\hline \multirow{2}{*}{\multicolumn{2}{|c|}{ NIVERSITY OF NEVADA, RENO }} & UNIVE & OF U & \\
\hline & & WASH & N ST & ERSIT \\
\hline \multicolumn{2}{|c|}{$\begin{array}{l}\text { NEW MEXICO STATE UNIVERSITY } \\
\text { OREGON STATE UNIVERSITY }\end{array}$} & UNIVERS & OF WASHI & \\
\hline
\end{tabular}

The Supporting Institutions listed above contribute to the cost of publication of this Journal, but they are not owners or publishers and have no responsibility for its content or policies.

Mathematical papers intended for publication in the Pacific Journal of Mathematics should be in typed form or offset-reproduced (not dittoed), double spaced with large margins. Please do not use built up fractions in the text of the manuscript. However, you may use them in the displayed equations. Underline Greek letters in red, German in green, and script in blue. The first paragraph must be capable of being used separately as a synopsis of the entire paper. In particular it should contain no bibliographic references. Please propose a heading for the odd numbered pages of less than 35 characters. Manuscripts, in triplicate, may be sent to any one of the editors. Please classify according to the 1991 Mathematics Subject Classification scheme which can be found in the December index volumes of Mathematical Reviews. Supply name and address of author to whom proofs should be sent. All other communications should be addressed to the managing editor, or Elaine Barth, University of California, Los Angeles, California 90024-1555-05.

There are page-charges associated with articles appearing in the Pacific Journal of Mathematics. These charges are expected to be paid by the author's University, Government Agency or Company. If the author or authors do not have access to such Institutional support these charges are waived. Single authors will receive 50 free reprints; joint authors will receive a total of 100 free reprints. Additional copies may be obtained at cost in multiples of 50 .

The Pacific Journal of Mathematics (ISSN 0030-8730) is published monthly except for July and August. Regular subscription rate: $\$ 190.00$ a year (10 issues). Special rate: $\$ 95.00$ a year to individual members of supporting institutions.

Subscriptions, orders for numbers issued in the last three calendar years, and changes of address should be sent to Pacific Journal of Mathematics, P.O. Box 969, Carmel Valley, CA 93924, U.S.A. Old back numbers obtainable from Kraus Periodicals Co., Route 100, Millwood, NY 10546.

The Pacific Journal of Mathematics at P.O. Box 969, Carmel Valley, CA 93924 (ISSN 0030-8730) is published monthly except for July and August. Second-class postage paid at Carmel Valley, California 93924, and additional mailing offices. Postmaster: send address changes to Pacific Journal of Mathematics, P.O. Box 969, Carmel Valley, CA 93924.

PUBLISHED BY PACIFIC JOURNAL OF MATHEMATICS, A NON-PROFIT CORPORATION Copyright (C) 1992 by Pacific Journal of Mathematics 


\section{Pacific Journal of Mathematics}

Vol. 153, No. $2 \quad$ April, 1992

R. Ayala, Eladio Domínguez Murillo, Alberto Márquez Pérez and A.

Quintero, Lusternik-Schnirelmann invariants in proper homotopy

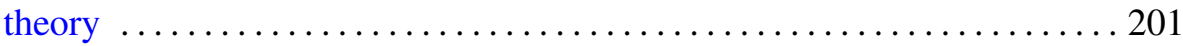

Hari Bercovici and Dan-Virgil Voiculescu, Lévy-Hinčin type theorems for multiplicative and additive free convolution $\ldots \ldots \ldots \ldots \ldots \ldots \ldots 217$

L. J. Bunce and Cho-Ho Chu, Compact operations, multipliers and Radon-Nikodým property in $J B^{*}$-triples $\ldots \ldots \ldots \ldots \ldots \ldots \ldots \ldots \ldots \ldots \ldots \ldots$

Marius Dadarlat, Gabriel Nagy, András Némethi and Cornel Pasnicu, Reduction of topological stable rank in inductive limits of $C^{*}$-algebras

François Dumas and Robert Vidal, Dérivations, et hautes dérivations, dans certains corps gauches de series de Laurent .................... 277

Mourad Ismail and Xin Li, On sieved orthogonal polynomials. IX:

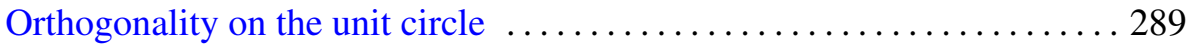

X. T. Liang and Y. W. Lu, A Phragmén-Lindelöf theorem ...............299

Mark Stephen Reeder, On certain Iwahori invariants in the unramified principal series

Shohei Tanaka, On the representation of the determinant of Harish-Chandra's $C$-function of $\operatorname{SL}(n, \mathbb{R})$

Fritz von Haeseler and Guentcho Svetoslavov Skordev, Borsuk-Ulam theorem, fixed point index and chain approximations for maps with multiplicity 